Comment. Math. Helv. 79 (2004) 704-752

0010-2571/04/040704-49

DOI $10.1007 / \mathrm{s} 00014-004-0818-9$ (c) 2004 Birkhäuser Verlag, Basel

Commentarii Mathematici Helvetici

\title{
Simultaneous metric uniformization of foliations by Riemann surfaces
}

\author{
A. A. Glutsyuk
}

\begin{abstract}
We consider a two-dimensional linear foliation on torus of arbitrary dimension. For any smooth family of complex structures on the leaves we prove existence of smooth family of uniformizing (conformal complete flat) metrics on the leaves. We extend this result to linear foliations on $\mathbb{T}^{2} \times \mathbb{R}$ and families of complex structures with bounded derivatives $C^{3}$-close to the standard complex structure. We prove that the analogous statement for arbitrary $C^{\infty}$ twodimensional foliation on compact manifold is wrong in general, even for suspensions over $\mathbb{T}^{2}$ : in dimension 3 the uniformizing metric can be nondifferentiable at some points; in dimension 4 the uniformizing metric of each noncompact leaf can be unbounded.
\end{abstract}

Mathematics Subject Classification (2000). 53C12, 30F10, 58F18.

Keywords. Foliation by Riemann surfaces, almost complex structure, uniformization, uniformizing metric.

\section{Introduction, statement of results and history}

\subsection{Introduction and brief statement of results}

The (almost) complex structure on a two-dimensional surface is a family of complex structures on the tangent planes at the points of the surface. We say that a (nonstandard) complex structure on a Riemann surface is bounded if it has uniformly bounded dilatation with respect to the standard complex structure.

It is well-known that each measurable bounded complex structure is locally integrable. This was proved in $[\mathrm{M}]$ and earlier under additional regularity conditions (Hölder or continuous) in [Ko], [Licht], [La]. Each measurable complex structure on $\mathbb{C}$ is globally integrable:

1.1. Theorem ([Ah1], $[\mathrm{AhB}],[\mathrm{Vek}],[\mathrm{M}])$. For any measurable $\left(C^{\infty}\right)$ bounded complex structure $\sigma$ on $\mathbb{C}$ there exists a quasiconformal homeomorphism $\left(C^{\infty}\right.$ diffeomorphism) $\mathbb{C} \rightarrow \mathbb{C}$ that transforms $\sigma$ to the standard complex structure. 
The definition of a quasiconformal homeomorphism may be found in [Ah2]. Theorem 1.1 implies that for any $C^{\infty}$ metric $g$ on $\mathbb{R}^{2}$ with bounded dilatation there exists a $C^{\infty}$ positive function $\phi: \mathbb{R}^{2} \rightarrow \mathbb{R}_{+}$such that the metric $\phi g$ is flat and complete (the function $\phi$ is unique up to multiplication by constant). This statement remains valid with $\mathbb{R}^{2}$ replaced by arbitrary parabolic Riemann surface. (Recall that a Riemann surface is said to be parabolic, if its universal covering equipped with the natural complex structure is conformally equivalent to $\mathbb{C}$.)

In the present paper we study the existence of foliated versions of Theorem 1.1. Namely, we consider a real two-dimensional foliation on a Riemann manifold $(M, g)$ (in most cases under consideration $M$ is compact). The metric $g$ induces a complex structure on each leaf. We suppose that it is parabolic. By the previous statement, on each leaf $L$ there exists a function $\phi: L \rightarrow \mathbb{R}_{+}$such that the metric $\phi g$ of the leaf $L$ is flat and complete. We study the following questions.

Question 1. Is it possible to find a $C^{\infty}$ function $\phi: M \rightarrow \mathbb{R}_{+}$such that the restriction to each leaf of the metric $\phi g$ be flat and complete?

Question 2. If yes, is it possible to find an Euclidean metric $g^{\prime}$ on the ambient manifold $M$ that coincides with $\phi g$ along the leaves, and for which each leaf be totally geodesic?

1.2. Example. Denote $\mathbb{T}^{n}=\mathbb{R}^{n} / 2 \pi \mathbb{Z}^{n}$. Consider a two-dimensional parallel plane foliation on $\mathbb{R}^{n}$. The standard projection $\mathbb{R}^{n} \rightarrow \mathbb{T}^{n}$ induces a foliation on the torus $\mathbb{T}^{n}$. This foliation is called linear. Take a (nonstandard) metric $g$ on $\mathbb{T}^{n}$ and consider the corresponding complex structures on the leaves. Then each leaf is parabolic, by Theorem 1.1 and since the metric $g$ has a bounded dilatation with respect to the standard Euclidean metric (by compactness argument).

The analogous questions were studied by A. Verjovsky [Ver], A. Candel and X. Gómez-Mont [CGM], A. Lins Neto ([Li1], [Li2]) for some holomorphic foliations with singularities by hyperbolic Riemann surfaces, and by A. Candel [Ca], who completely answered the analogue of Question 1 for laminations by hyperbolic (elliptic) Riemann surfaces. In 1995 É. Ghys [G] had studied the (parabolic) case of linear foliations on $\mathbb{T}^{3}$ with a nonstandard metric $g$. He proposed Question 1 in the general case and he proved a positive answer for linear foliations on $\mathbb{T}^{3}$ under certain Diophantine condition on the slope of the leaves.

1.3. Remark. For any linear foliation on $\mathbb{T}^{n}$ either all the leaves are tori, or each leaf is dense. If the leaves are dense, then all they are either planes, or cylinders. In the simplest case, when the leaves of the foliation $F$ are tori, the positive answer to Question 1 for arbitrary metric $g$ follows from the classical theorem on uniformization of torus with variable complex structure [Ab], [Ah2] (Theorem 2.1 in Section 2).

Theorem 1.10 of the present paper gives a positive answer to Question 1 for arbitrary linear foliation on $\mathbb{T}^{n}$ in the cases of analytic, $C^{\infty}$ and measurable metric. 
In fact, we prove a stronger Theorem 1.12 on existence of a family of uniformizing holomorphic differentials on the leaves, see the following definition.

1.4. Definition. A holomorphic 1-form on a parabolic Riemann surface is said to be a uniformizing differential, if its squared module is a complete flat metric. A complete conformal flat metric on a parabolic Riemann surface is called a uniformizing metric.

1.5. Remark. A uniformizing metric (differential) exists and is unique up to multiplication by constant (for the statement on the uniformizing metric see the beginning of the paper). The squared module of a holomorphic differential is always a flat metric, thus, that of a uniformizing differential is a uniformizing metric. (This together with the uniqueness of the uniformizing metric and Liouville's theorem on bounded holomorphic functions implies the uniqueness of uniformizing differential.) Thus, by uniqueness, a uniformizing metric is always the squared module of a uniformizing differential.

To construct a family of holomorphic differentials, we use the homotopy method, which reduces the proof to solving a linear bounded ordinary differential equation in the space $L_{2}\left(\mathbb{T}^{n}\right)$. We prove regularity of its solution by showing that the equation is bounded in any Sobolev space $H^{s}\left(\mathbb{T}^{n}\right)$. The method of the proof yields a new short proof of the global integrability of a $C^{\infty}$ complex structure on $\mathbb{T}^{2}$ (Theorem 2.1 in Section 2). This together with the classical normality argument (using Grötzsch inequality [Ah2]) yields a new short proof of the general measurable case of Theorem 1.1 (to appear in [Gl]).

Another short proof of Theorem 1.1 using a different method (Fourier transformation) was earlier obtained by A. Douady and X. Buff [DB].

For the proof of existence of smooth family of uniformizing differentials (metrics) on the leaves, we forget the initial metric $g$ and consider only the corresponding (smooth) family of complex structures on the leaves. We do this without changing the context, by the following proposition.

1.6. Proposition. For any two-dimensional $C^{\infty}$ foliation any $C^{\infty}$ Riemann metric on the foliated manifold induces a $C^{\infty}$ family of complex structures on the leaves (by taking the conformal classes of its restrictions to the leaves). Conversely, each $C^{\infty}$ family of complex structures on the leaves is defined in this way by some $C^{\infty}$ metric on the manifold.

Proof. The first statement of the proposition follows from definition. The converse statement means that for any $C^{\infty}$ family of complex structures on the leaves there exists a $C^{\infty}$ metric on the foliated manifold whose restriction to each leaf is conformal. The local version of this statement follows from definition: it is obvious in the case of trivial parallel plane fibration of a cube. Its global version is proved by pasting together the local conformal metrics using a splitting of unity. 
Theorems 1.20 and 1.21 in Subsection 1.3 give a complete answer to Question 2 in the case of linear foliations on $\mathbb{T}^{n}$ for smooth and analytic metrics $g$. They say that if the slope of a foliation satisfies certain Diophantine condition, then there exists an Euclidean metric $g^{\prime}$ on $\mathbb{T}^{n}$ of the same regularity, as the initial metric $g$ that is equal to $\phi g$ along the leaves and for which all the leaves are totally geodesic. The Diophantine condition depends on the regularity class under consideration and is optimal.

Earlier A. Haefliger $[\mathrm{H}]$ have obtained a result implying that under an a priori stronger Diophantine condition the metric $\phi g$ extends up to a global metric on the torus for which all the leaves are minimal surfaces.

Theorem 1.23 in Subsection 1.4 gives a positive answer to Question 1 for any linear foliation on $\mathbb{T}^{2} \times \mathbb{R}$ and arbitrary metric $C^{3}$-close enough to the standard Euclidean metric and having uniformly bounded derivatives of each order.

Theorems 1.25 and 1.27 in Subsection 1.5 give nonlinear counterexamples to Question 1. The first counterexample (Theorem 1.25) is given by an analytic foliation on $\mathbb{T}^{3}$ that is a suspension of a circle diffeomorphism with two fixed points (attractor and repeller). This foliation has two toric leaves, the other ones are cylinders. We show that there exists an analytic metric on $\mathbb{T}^{3}$ such that the corresponding function $\phi$ (for which the restriction to each leaf of the metric $\phi g$ is flat) is analytic on the complement of the two toric leaves and nowhere differentiable in some toric leaf. The proof uses essentially an explicit formula for normalized uniformizing differentials corresponding to linear foliations on $\mathbb{T}^{2} \times \mathbb{R}$ (Proposition 5.8). The second counterexample (Theorem 1.27) is given by a smooth foliation on $\mathbb{T}^{2} \times S^{2}$ whose leaves are locally 1-to-1 projected to $\mathbb{T}^{2}$ : it is a suspension of a pair of commuting diffeomorphisms over the translations by the vectors $(1,0)$ and $(0,1)$. We construct a smooth metric on the foliated space such that the function $\phi$ corresponding to each noncompact leaf tends to infinity, as the point where it is taken tends to infinity along the leaf.

É. Ghys have noticed in 1995 [G] that Reeb foliation of the three-sphere does not admit a bounded complete conformal flat metric along the leaves, for arbitrary choice of the initial metric $g$. In the previous counterexample this is not true. Namely, there is a metric $g$ whose restriction to each leaf coincides with the lifting to the leaves of the Euclidean metric of $\mathbb{T}^{2}$. This metric itself is flat along the leaves.

The basic definitions concerning complex structures are given in 1.2. Theorems 1.12 and 2.1 are proved in Section 2. Theorems 1.20 and 1.21 are proved in Section 3. Theorems 1.23, 1.25, 1.27 are proved respectively in Sections 4, 5, 6 .

\subsection{Complex structures and uniformizing differentials. Basic notations}

Recall the following standard notations. To a (nonstandard almost) complex structure on a subset $D \subset \mathbb{C}$ we put into correspondence the $\mathbb{C}$-valued 1-form of the type

$$
\omega_{\mu}=d z+\mu(z) d \bar{z},|\mu|<1,
$$


that is $\mathbb{C}$-linear on each tangent plane with respect to the chosen complex structure. The function $\mu$ is uniquely defined. Vice versa, for arbitrary complex-valued function $\mu,|\mu|<1$, the 1-form (1.1) defines the unique complex structure for which it is $\mathbb{C}$-linear. The complex structure is bounded, if and only if $\sup |\mu|<1$.

1.7. Remark. If the form (1.1) defines a bounded complex structure on $\mathbb{C}$, then the corresponding uniformizing differential is a

closed form $f \omega_{\mu}, f: \mathbb{C} \rightarrow \mathbb{C} \backslash 0$, such that $\left|f \omega_{\mu}\right|^{2}$ is a complete metric.

Vice versa, any form (1.2) is always a uniformizing differential for the corresponding complex structure.

1.8. Remark. Quasiconformal homeomorphisms $\Phi: \mathbb{C} \rightarrow \mathbb{C}$ act on the space of complex structures on $\mathbb{C}$ : the pull-back (denoted $\sigma$ ) of the standard complex structure under a homeomorphism $\Phi$ is defined by the function $\mu=\frac{\partial \Phi}{\partial \bar{z}} / \frac{\partial \Phi}{\partial z}$. The differential $d \Phi$ is a uniformizing differential corresponding to the nonstandard complex structure $\sigma$. Theorem 1.1 recalled in the introduction says that each complex structure on $\mathbb{C}$ defined by a measurable function $\mu$ with $\sup |\mu|<1$ can be transformed to the standard one by appropriate quasiconformal homeomorphism.

We consider two-dimensional foliations on products $M \times \mathbb{T}^{2}$ that are suspensions, i.e., whose leaves are locally 1-to-1 projected to $\mathbb{T}^{2}$. Let $z$ be the standard complex coordinate on $\mathbb{T}^{2}$. It induces a local complex coordinate on each leaf (also denoted by $z$ ) by lifting.

1.9. Definition. The standard complex structure on leaves of an above foliation is the lifting of the standard complex structure of $\mathbb{T}^{2}$.

Each function $\mu: M \times \mathbb{T}^{2} \rightarrow \mathbb{C}$, sup $|\mu|<1$, defines a family of bounded complex structures on the leaves so that the corresponding form (1.1) is $\mathbb{C}$-linear on the tangent planes to the leaves, and vice versa. We say that a family of complex structures is smooth (analytic, etc.), if so is the corresponding function $\mu$.

Let $f: M \times \mathbb{T}^{2} \rightarrow \mathbb{C} \backslash 0$ be a function. The condition that the 1-form $f \omega_{\mu}$ is closed along the leaves (a necessary condition to be a uniformizing differential along the leaves) may be written explicitly as a PDE on the function $f$. Namely, consider the following differential operators acting on functions on the foliated manifold:

$$
D_{z}=\frac{\partial}{\partial z}, D_{\bar{z}}=\frac{\partial}{\partial \bar{z}}, \text { both taken along the leaves with the local coordinate } z \text {. }
$$

Then a 1-form $f \omega_{\mu}$ is closed along each leaf, if and only if

$$
D_{\bar{z}} f=D_{z}(\mu f) \text {. }
$$




\subsection{Uniformizability of linear foliations}

1.10. Theorem. Let $F$ be arbitrary linear foliation on $\mathbb{T}^{n}, g$ be a Riemann metric on $\mathbb{T}^{n}$ that is analytic (respectively, $C^{\infty}$ /measurable uniformly bounded from below with uniformly bounded dilatation along the leaves of $F$ ). There exists an analytic (respectively, $C^{\infty} / L_{1}$ ) positive function $\phi: \mathbb{T}^{n} \rightarrow \mathbb{R}_{+}$such that the restriction of the metric $\phi g$ to each leaf (almost each in the measurable case) of the foliation $F$ is uniformizing: it is flat (in the sense of distributions in the third case) and complete.

1.11. Remark. In the previous theorem in the smooth and analytic cases the completeness of the metric $\phi g$ follows from the nonvanishing of the function $f$ and compactness argument.

We prove the following stronger theorem.

1.12. Theorem. Let $F$ be a linear foliation on $\mathbb{T}^{n}, \sigma$ be a family of almost complex structures on the leaves of $F$ that is analytic (respectively, $C^{\infty} /$ measurable with uniformly bounded dilatation) on $\mathbb{T}^{n}$. There exists a nowhere vanishing analytic (respectively, $C^{\infty} / L_{2}$ ) differential 1-form $\omega$ on $\mathbb{T}^{n}$ whose restriction to each leaf is a uniformizing differential with respect to the complex structure $\sigma$. The form $\omega$ can be normalized to depend analytically on the complex-valued functional parameter $\mu$ of $\sigma$ (see the previous subsection).

Theorem 1.12 is proved in Subsections $2.1\left(C^{\infty}\right.$ case) and 2.2 (analytic and measurable cases). The squared modules of the uniformizing differentials from Theorem 1.12 are the uniformizing metrics from Theorem 1.10.

1.13. Remark. If in the conditions of the previous theorem the leaves of the foliation are dense, then the corresponding function $f$ is unique up to multiplication by constant. Indeed uniqueness of uniformizing differential applied leafwise implies that the function $f$ is unique up to multiplication by a function whose restriction to almost each leaf is constant. Density of the leaves implies that the latter miltiplier function is constant almost everywhere.

1.14. Corollary. In the conditions of the previous theorem the universal covering of each leaf (almost each in the measurable case) equipped with the standard complex structure (induced by the Euclidean metric) admits a quasiconformal homeomorphism onto $\mathbb{C}$ whose derivative is the pullback of the form $\omega$ under the covering projection (in the sense of distributions in the measurable case).

The corollary follows from Theorems 1.1, 1.12 and the uniqueness of uniformizing differential (see Remark 1.5). 


\subsection{Existence of conformal Euclidean metric for which leaves are totally geodesic}

Below we formulate the statement from the title of the subsection for linear foliations on $\mathbb{T}^{n}$ with nonstandard metric $g$ under appropriate Diophantine conditions on the slope of the foliation. They are two different conditions (see Definition 1.16) corresponding to the cases, when the initial metric $g$ is smooth (respectively, analytic).

1.15. Definition. We say that a number $\alpha \in \mathbb{R} \backslash \mathbb{Q}$ is Diophantine, if there exist constants $C>0, s \geq 1$ such that for any pair $m, k \in \mathbb{Z}, k \neq 0$, the following inequality holds:

$$
\left|\alpha-\frac{m}{k}\right|>\frac{C}{|k|^{s+1}}
$$

1.16. Definition. Consider a foliation on $\mathbb{R}^{n}$ by parallel planes: level planes of a linear vector-function of rank $n-2$. Let $W$ be the $n-2$-space passing through the origin and orthogonal to the planes. Let $F$ be the corresponding factorized linear foliation on $\mathbb{T}^{n}$. Say that $F$ is Diophantine, if there exist constants $C>0$, $s \geq 1$ such that for any $N=\left(N_{1}, \ldots, N_{n}\right) \in \mathbb{Z}^{n} \backslash 0$

$$
\operatorname{dist}(N, W)>\frac{C}{|N|^{s}}, \quad|N|=\sum_{i}\left|N_{i}\right|
$$

Say that $F$ is weakly Diophantine, if

$$
\underline{\lim }_{N \in \mathbb{Z}^{n},|N| \rightarrow \infty}(\operatorname{dist}(N, W))^{\frac{1}{|N|}}=1 .
$$

1.17. Remark. Let $n=3, x=\left(x_{1}, x_{2}, x_{3}\right)$ be coordinates in the space $\mathbb{R}^{3}$. Consider the foliation on $\mathbb{R}^{3}$ by level planes of the linear function $l(x)=a_{1} x_{1}+$ $a_{2} x_{2}-x_{3}$. Then the corresponding linear foliation $F$ on $\mathbb{T}^{3}$ is Diophantine, if and only if there exist constants $C>0, s \geq 1$ such that for any $N=\left(N_{1}, N_{2}, N_{3}\right) \in$ $\mathbb{Z}^{3} \backslash 0$ the following inequality holds:

$$
\left|N_{1}+a_{1} N_{3}\right|+\left|N_{2}+a_{2} N_{3}\right|>\frac{C}{|N|^{s}}, \quad|N|=\left|N_{1}\right|+\left|N_{2}\right|+\left|N_{3}\right| .
$$

It is weakly Diophantine, if and only if

$$
\underline{\lim }_{N \in \mathbb{Z}^{3},|N| \rightarrow \infty}\left(\left|N_{1}+a_{1} N_{3}\right|+\left|N_{2}+a_{2} N_{3}\right|\right)^{\frac{1}{|N|}}=1 .
$$

1.18. Example. In the notations of the previous remark let the additive subgroup in $\mathbb{R}$ generated by $a_{1}$ and $a_{2}$ contain a Diophantine number. Then the foliation $F$ is Diophantine. It is not known to the author, whether the converse is true. 
1.19. Remark. The limit in (1.6) is always less than or equal to 1. A Diophantine foliation is always weakly Diophantine.

1.20. Theorem. Let $F$ be a Diophantine foliation (see Definition 1.16), $g$ be a $C^{\infty}$ metric on $\mathbb{T}^{n}$, $\sigma$ be the family of complex structures on the leaves of $F$ such that $g$ is $\sigma$-conformal. There exists a $C^{\infty}$ Euclidean metric on $\mathbb{T}^{n}$ such that

all the leaves are totally geodesic and its restriction

to each leaf is $\sigma$-conformal.

Or equivalently, there exist a discrete rank $n$ additive subgroup $G \subset \mathbb{R}^{n}$ and $\left(C^{\infty}\right)$ diffeomorphism $\mathbb{T}^{n} \rightarrow \mathbb{T}_{G}=\mathbb{R}^{n} / G$ that transforms $F$ to a linear foliation and $\sigma$ to the standard complex structure induced by the standard Euclidean metric. Conversely, if a linear foliation on $\mathbb{T}^{n}$ is not Diophantine, then there exists a $C^{\infty}$ metric $g$ on the torus such that there is no $C^{2}$ Euclidean metric on $\mathbb{T}^{n}$ satisfying (1.7).

1.21. Theorem. Let $F$ be a weakly Diophantine foliation (see Definition 1.16). Then for any analytic metric $g$ on $\mathbb{T}^{n}$ there exists an analytic Euclidean metric on $\mathbb{T}^{n}$ that satisfies (1.7). Conversely, if $F$ is not weakly Diophantine, then there exists an analytic metric $g$ on $\mathbb{T}^{n}$ such that there is no $C^{2}$ Euclidean metric on $\mathbb{T}^{n}$ that satisfies (1.7).

Theorems 1.20 and 1.21 are proved in Section 3.

Let us justify the equivalence of the two statements of Theorem 1.20. Clearly the second one implies the first one: the Euclidean metric from the first statement is the pull-back of the standard one under the diffeomorphism from the second statement. Let us prove the converse. Any Euclidean metric on a torus is transformed under appropriate diffeomorphism into the standard Euclidean metric on another torus (that is a quotient of the space by another lattice in general). Consider the images of leaves of the foliation. Their liftings to the space are planes, since the leaves are totally geodesic. They are parallel: the liftings to the space of any two leaves of the initial foliation remain on a bounded distance from each other; therefore, the same is true for the liftings of their images, hence, they are parallel. Therefore, the leaves are transformed to the leaves of a linear foliation. This proves that statement (1.7) of Theorem 1.20 implies its second statement.

\subsection{Uniformizability of linear folations on $\mathbb{T}^{2} \times \mathbb{R}$}

Below we formulate the analogue of Theorem 1.12 for linear foliations on $\mathbb{T}^{2} \times \mathbb{R}$, on existence of family of uniformizing metrics on the leaves for any $C^{\infty}$-family of complex structures on the leaves $C^{3}$-close to the standard one with bounded derivatives of all orders. 
1.22. Remark. A linear foliation on $\mathbb{T}^{2} \times \mathbb{R}$ is defined analogously to that on a torus. Its leaves are either all tori, or all cylinders, or all planes. In the case, when they are planes (and only in this case) they are dense. If they are tori or cylinders, then for any $C^{\infty}$ metric $g$ on $\mathbb{T}^{2} \times \mathbb{R}$ there exists a $C^{\infty}$ function $\phi: \mathbb{T}^{2} \times \mathbb{R} \rightarrow \mathbb{R}_{+}$ such that the restriction to each leaf of the metric $\phi g$ be flat and complete (see Remark 1.3 in the case of tori and Lemma 5.4 (in Subsection 5.1) in the case of cylinders).

Everywhere below without loss of generality we consider that the leaves of a linear foliation on $\mathbb{T}^{2} \times \mathbb{R}$ under consideration are locally 1-to-1 projected to $\mathbb{T}^{2}$ : one can achieve this by applying appropriate affine automorphism of $\mathbb{T}^{2} \times \mathbb{R}$.

Denote $C_{b}^{\infty}\left(\mathbb{T}^{2} \times \mathbb{R}\right)$ the space of bounded $C^{\infty}$ functions on $\mathbb{T}^{2} \times \mathbb{R}$ with bounded derivatives of all orders. For any $f \in C_{b}^{\infty}\left(\mathbb{T}^{2} \times \mathbb{R}\right)$ and any $r \in \mathbb{N} \cup 0$ define

$$
\begin{aligned}
\|f\|_{C^{r}} & =\sum_{\alpha=\left(\alpha_{1}, \alpha_{2}, \alpha_{3}\right) \in(\mathbb{N} \cup 0)^{3}, 0 \leq|\alpha| \leq r} \sup _{x \in \mathbb{T}^{2} \times \mathbb{R}}\left|\partial_{\alpha} f\right|(x) ;|\alpha| \\
& =\left|\alpha_{1}\right|+\left|\alpha_{2}\right|+\left|\alpha_{3}\right| .
\end{aligned}
$$

Let $x_{1}, x_{2}$ be the standard coordinates on $\mathbb{T}^{2}, s$ be an affine coordinate on $\mathbb{R}$.

1.23. Theorem. There exists a $\delta>0$ satisfying the following statement. Let $F$ be a linear foliation on $\mathbb{T}^{2} \times \mathbb{R}, \sigma$ be a $C^{\infty}$ family of complex structures on the leaves of $F, \mu: \mathbb{T}^{2} \times \mathbb{R} \rightarrow \mathbb{C}$ be the corresponding function from (1.1). Let $\mu$ have bounded derivatives of each order, and

$$
\|\mu\|_{C^{3}}<\delta .
$$

Then the foliation by Riemann surfaces defined by $F$ and $\sigma$ admits a $C_{b}^{\infty}$ family of uniformizing differentials, see Definition 1.4 (and hence, a $C_{b}^{\infty}$ family of uniformizing metrics) on the leaves. The previous statement holds for $\delta=\frac{1}{580}$. The corresponding family of uniformizing differentials can be normalized to depend analytically on the complex-valued functional parameter $\mu$.

Addendum to Theorem 1.23. Let in Theorem 1.23 the function $\mu$ extend holomorphically to a complex neighborhood $\left|\operatorname{Im} x_{1}\right|+\left|\operatorname{Im} x_{2}\right|+|\operatorname{Im} s|<\Delta, \Delta>0$, of the real space $\mathbb{T}^{2} \times \mathbb{R}$ so that the restriction of $\mu$ to each fiber $T_{y_{1}, y_{2}, y_{3}}=\left\{\operatorname{Im} x_{1}=\right.$ $\left.y_{1}, \operatorname{Im} x_{2}=y_{2}, \operatorname{Im} s=y_{3}\right\}$ has $C^{3}$-norm less than $\delta$. Then the corresponding families of uniformizing differentials and metrics are analytic on $\mathbb{T}^{2} \times \mathbb{R}$.

Theorem 1.23 and the Addendum are proved in Section 4.

1.24. Remark. It is not known, whether the statements of Theorem 1.23 remain valid with inequality (1.9) replaced just by the condition $\sup |\mu|<1$ of boundedness of the dilatation of the complex structure $\sigma$. 


\subsection{Nonuniformizability. Counterexamples to Question 1}

Here we state the Theorems on existence of the counterexamples to Question 1 mentioned in the Introduction.

1.25. Theorem. There exists a two-dimensional analytic foliation $F$ on $\mathbb{T}^{3}=$ $\mathbb{T}^{2} \times S^{1}$ with the following properties.

1) $F$ is invariant under the translations of $\mathbb{T}^{2}$.

2) Any leaf is locally 1-to-1 projected to $\mathbb{T}^{2}$.

3) There are exactly two leaves homeomorphic to tori; any other leaf is homeomorphic to the cylinder $S^{1} \times \mathbb{R}$.

4) There exists an analytic family of complex structures on the leaves satisfying the two following statements:

a) there is a unique continuous family of uniformizing metrics on the leaves up to multiplication by constant; it is analytic outside the previous toric leaves;

b) the latter family of uniformizing metrics is not differentiable in the transversal parameter at one of the toric leaves.

1.26. Remark. In fact, the foliation and the family of complex structures from Theorem 1.25 constructed in Section 5 satisfy the following additional statement: the corresponding families of uniformizing differentials and metrics are analytic in the whole complement to the toric leaf of nondifferentiability (see statement $4 \mathrm{~b}$ ), including the other toric leaf. This statement will not be used further and its proof is omitted to save the space. One can prove it by using the results of Section 5.

1.27. Theorem. There exists a two-dimensional $C^{\infty}$ foliation $F$ on $\mathbb{T}^{2} \times S^{2}$ with the following properties.

1) $F$ is invariant under the translations of $\mathbb{T}^{2}$.

2) Any leaf is locally 1-to-1 projected to $\mathbb{T}^{2}$.

3) There is a big circle $S^{1} \subset S^{2}$ such that the product $\mathbb{T}^{2} \times S^{1}$ is the union of leaves of $F$; each of these leaves is a horizontal torus $\mathbb{T}^{2} \times a, a \in S^{1}$.

4) Any other leaf is diffeomorphic to $\mathbb{R}^{2}$ and its accumulation set is the previous product $\mathbb{T}^{2} \times S^{1}$.

5) There exists a $C^{\infty}$ family of complex structures on the leaves such that the uniformizing metric (denoted UM) of each noncompact leaf $L$ (which accumulates to $\mathbb{T}^{2} \times S^{1}$ ) is unbounded: more precisely, for given a $C^{\infty}$ metric $H$ on $\mathbb{T}^{2} \times S^{2}$ and a noncompact leaf $L$ the ratio $\frac{U M}{\left.H\right|_{L}}(x)$ tends to infinity, as $x \rightarrow \mathbb{T}^{2} \times S^{1}, x \in L$.

Theorem 1.25 is proved in Section 5. Theorem 1.27 is proved in Section 6 . 


\section{Uniformization of linear foliations on tori: proof of Theo- rem 1.12. Uniformization of torus with variable complex struc-} ture

We prove the $C^{\infty}$ version of Theorem 1.12 in Subsection 2.1 and the analytic and measurable versions in Subsection 2.2 (the proofs are analogous). For simplicity we present the proofs only in the case, when $n=3$. The proofs remain valid in higher dimensions with obvious changes. Everywhere in Subsections 2.1, 2.2 we consider that the leaves of the linear foliation $F$ on $\mathbb{T}^{3}$ under consideration are dense (see Remark 1.3).

In Subsection 2.4 we give a proof of the following version of Theorem 1.1 for torus with variable $C^{\infty}$ complex structure. The method of the proof is the same as the one of the proof of Theorem 1.12.

2.1. Theorem $([\mathrm{Ab}])$. Let $\mu: \mathbb{T}^{2} \rightarrow \mathbb{C}$ be a $C^{\infty}$ function, $|\mu|<1$. Then there exists a nonvanishing $C^{\infty}$ function $f: \mathbb{T}^{2} \rightarrow \mathbb{C} \backslash 0$ such that the form $f \omega_{\mu}=$ $f(d z+\mu d \bar{z})$ is closed (and hence, is a uniformizing differential). The function $f$ normalized to have unit average along a given noncontractible closed curve depends analytically on the complex-valued function $\mu$.

Theorem 2.1 is proved in Subsection 2.3. In the proof of Theorems 1.12 and 2.1 we use the local integrability of a $C^{\infty}$ complex structure (see [Ko], [Licht], [La] and Proposition 2.4 stated below). Its independent proof is given in Subsection 2.4.

\subsection{Homotopy method. Proof of Theorem 1.12 in the $C^{\infty}$ case}

We use the notations of Remark 1.17: the coordinates $\left(x_{1}, x_{2}, x_{3}\right)$ on $\mathbb{T}^{3}$ are chosen so that the $x_{3}$-axis is transversal to the lifting to the space of the foliation $F$. Define the complex coordinate $z=x_{1}+i x_{2}$ on the leaves of the foliation $F$ and on those of its lifting (the coordinates $x_{i}, z$ and their projection pushforwards to the torus will be denoted by the same symbols $x_{i}$ and $z$ ).

Let $\mu: \mathbb{T}^{3} \rightarrow \mathbb{C}$ be the function defining the complex structure $\sigma$ on the leaves (see (1.1)). To construct a $C^{\infty}$ family of uniformizing differentials from Theorem 1.12 , it suffices to construct a $C^{\infty}$ function $f: \mathbb{T}^{3} \rightarrow \mathbb{C} \backslash 0$ such that the restriction to the leaves of the form $\omega=f \omega_{\mu}$ is closed (see the end of Subsection 1.2 and Remark 1.11). To do this, we use the homotopy method. Namely, we include the complex structure $\sigma$ into the one-parametric family of complex structures (denoted by $\sigma_{\nu}$ ) defined by their $\mathbb{C}$-linear 1 -forms

$$
\omega_{\nu}=d z+\nu(x, t) d \bar{z}, \nu(x, t)=t \mu(x), t \in[0,1] .
$$

The complex structure on the leaves that corresponds to the parameter value $t=0$ is the standard one, the given structure $\sigma=\sigma_{\mu}$ corresponds to the value $t=1$. We will find a $C^{\infty}$ family $f(x, t): \mathbb{T}^{3} \times[0,1] \rightarrow \mathbb{C} \backslash 0$ of complex-valued nowhere 
vanishing $C^{\infty}$ functions on $\mathbb{T}^{3}$ depending on the same parameter $t, f(x, 0) \equiv$ 1 , such that the differential forms $f(x, t) \omega_{\nu}$ are closed on the leaves. Then the function $f=f(x, 1)$ is the one we are looking for.

The possibility to choose it analytic in $\mu$ (the last statement of Theorem 1.12) will be proved at the end of the subsection.

To construct the previous family of functions, we will find firstly a family $f(x, t)$ of nonidentically-vanishing (not necessarily nonvanishing) functions such that the forms $f \omega_{\nu}$ are closed on the leaves (the next lemma). Then we show that in fact, the functions $f(x, t)$ vanish nowhere (the next proposition): this will prove the first statement of Theorem 1.12. To do this (and only in this place) we use the local integrability of a $C^{\infty}$ complex structure (Proposition 2.4).

2.2. Lemma. Let $F$ be a linear foliation on $\mathbb{T}^{3}, \nu(x, t): \mathbb{T}^{3} \times[0,1] \rightarrow \mathbb{C}$ be a $C^{\infty}$ (analytic) family of $C^{\infty}$ functions on $\mathbb{T}^{3}$ depending on the parameter $t \in[0,1]$, $\nu(x, 0) \equiv 0,|\nu|<1$. There exists a $C^{\infty}$ (analytic) family $f(x, t)$ of complexvalued $C^{\infty}$ functions on $\mathbb{T}^{3}$ depending on the same parameter $t, f(x, 0) \equiv 1$, that do not vanish identically and such that the restrictions to the leaves of the form $f(x, t)(d z+\nu(x, t) d \bar{z})$ are closed.

The lemma is proved below.

2.3. Proposition. In the conditions of Lemma 2.2 the functions $f(x, t)$ vanish nowhere, if the leaves are dense.

Proof. Let us prove that $f(x, t) \neq 0$ by contradiction. Suppose the contrary. Then the set of the parameter values $t$ corresponding to the functions $f(x, t)$ on the torus having zeroes is nonempty (denote this set by $M$ ). Its complement $[0,1] \backslash M$ is open by definition. Let us show that the set $M$ is open as well. This will imply that the parameter segment is a union of two disjoint open sets, which will bring us to contradiction. It suffices to show that the (local) presence of a zero of a function $f$ persists under perturbation. To do this, we use the following

2.4. Proposition ([Ko], [Licht], [La]). Let $\mu: D \rightarrow \mathbb{C}$ be a $C^{\infty}$ function on a disc $D \subset \mathbb{C}$ centered at $0,|\mu|<1$. Then there exists a local univalent complex coordinate $w$ on a neighborhood of zero that is holomorphic with respect to the complex structure defined by $\mu$ (see (1.1)).

An independent proof of Proposition 2.4 will be given in Subsection 2.4.

Suppose $f\left(x_{0}, t\right)=0$ for some $x_{0}, t$ (let us fix them, denote $L_{x_{0}}$ the leaf passing through $x_{0}$ ). Let $w$ be the local holomorphic coordinate on $L_{x_{0}}$ from the previous proposition corresponding to $\mu=\nu(x, t), w\left(x_{0}\right)=0$. Suppose that the function $f(x, t)$ does not vanish identically on $L_{x_{0}}$ locally near $x_{0}$ : one can achieve this by choosing appropriate $x_{0}$, since $f$ does not vanish identically and the leaves are 
dense. Then $f \omega_{\mu}=\left(w^{k}+\right.$ higher terms $) d w$ by holomorphy of $w$ and $f \omega_{\mu}, k \geq 1$. Now by the index argument, the local presence of zero of $f$ on $L_{x_{0}}$ persists under perturbation. This together with the previous discussion proves the inequality $f(x, t) \neq 0$ and Proposition 2.3 modulo Proposition 2.4

By the discussion preceding the previous lemma, the lemma and Proposition 2.3 imply Theorem 1.12 (modulo the analytic dependence on $\mu$, which will be proved at the end of the subsection).

Proof of Lemma 2.2. We construct a family $f(x, t)$ from the lemma as a solution of a linear ordinary bounded differential equation in $L_{2}\left(\mathbb{T}^{3}\right)$. We prove regularity of its solutions by showing that it is bounded also in each Sobolev space $H^{s}\left(\mathbb{T}^{3}\right)$.

Recall that the condition that the form $f \omega_{\nu}$ is closed on the leaves is equivalent to the equation (1.4):

$$
D_{\bar{z}} f=D_{z}(\nu f), D_{z}, D_{\bar{z}} \text { are the differential operators from (1.3). }
$$

Differentiating it in $t$ and writing "dot" instead of the operator $\frac{\partial}{\partial t}$ yields

$$
D_{\bar{z}} \dot{f}-\left(D_{z} \circ \nu\right) \dot{f}=\left(D_{z} \circ \dot{\nu}\right) f
$$

where $D_{z} \circ \nu\left(D_{z} \circ \dot{\nu}\right)$ is the composition of the operator of the multiplication by the function $\nu$ (respectively, $\dot{\nu}$ ) and the operator $D_{z}$. Any solution $f$ of equation (2.2) with the initial condition $f(x, 0) \equiv 1$ that vanishes identically on the torus for no value of $t$ is a one we are looking for. Let us show that (2.2) is implied by a bounded linear differential equation in $L_{2}\left(\mathbb{T}^{3}\right)$. To do this, we use the following properties of the operators $D_{z}$ and $D_{\bar{z}}$ in the case of linear foliation.

2.5. Remark. The operators $D_{z}$ and $D_{\bar{z}}$ from (1.3) corresponding to a linear foliation on $\mathbb{T}^{3}$ have the common eigenfunctions $e^{i(N, x)}, N=\left(N_{1}, N_{2}, N_{3}\right) \in \mathbb{Z}^{3}$ (which form an orthogonal base in the space $L_{2}\left(\mathbb{T}^{3}\right)$ ). The corresponding eigenvalues (denote them respectively by $\lambda_{N}$ and $\lambda_{N}^{\prime}$ ) have equal modules, more precisely,

$$
\lambda_{N}^{\prime}=-\bar{\lambda}_{N}
$$

This is implied by the fact that the operator $D_{\bar{z}}$ is conjugated to $-D_{z}$ in the $L_{2}$ scalar product, which follows from definition. If the foliation $F$ under consideration is a pushforward of the level plane foliation for a linear function $l(x)=a_{1} x_{1}+$ $a_{2} x_{2}-x_{3}, a=a_{1}+i a_{2}$, then

$$
\begin{aligned}
D_{z}=\frac{\partial}{\partial z}+\frac{\bar{a}}{2} \frac{\partial}{\partial x_{3}}, \quad D_{\bar{z}} & =\frac{\partial}{\partial \bar{z}}+\frac{a}{2} \frac{\partial}{\partial x_{3}}, a=a_{1}+i a_{2} ; \text { therefore, } \\
\lambda_{N} & =\frac{i}{2}\left(N_{1}-i N_{2}+N_{3} \bar{a}\right) .
\end{aligned}
$$

This implies that the leaves of the foliation $F$ are dense, if and only if $\lambda_{N} \neq 0$ for all $N \neq 0$. 
2.6. Corollary. For any linear foliation $F$ on $\mathbb{T}^{3}$ there exists a unitary operator $U: L_{2}\left(\mathbb{T}^{3}\right) \rightarrow L_{2}\left(\mathbb{T}^{3}\right)$ preserving averages and the spaces of $C^{\infty}$ (analytic) functions such that " $U=D_{\bar{z}}^{-1} \circ D_{z}$ " (more precisely, $U \circ D_{\bar{z}}=D_{\bar{z}} \circ U=D_{z}$ ). This operator is unique, if and only if the leaves of the foliation $F$ are dense. The operator $U$ may be always chosen to commute with partial differentiations and to extend up to a unitary operator to any Hilbert-Sobolev space of functions on $\mathbb{T}^{3}$.

The operator $U$ from the previous corollary is defined to have the eigenfunctions $e^{i(N, x)}$; the corresponding eigenvalue is equal to $\frac{\lambda_{N}}{\lambda_{N}^{\prime}}=-\frac{\lambda_{N}}{\lambda_{N}}($ see $(2.3))$ if $\lambda_{N} \neq 0$ and to 1 otherwise, i.e., if $D_{z} e^{i(N, x)}=0$ (in this case it could be taken to be arbitrary number with unit module, if $N \neq 0$ ). The fact that $U$ preserves the space of analytic functions follows from their characterization (3.6) in terms of growth rate of Fourier coefficients.

Let us write down equation (2.2) in terms of the new operator $U$. Applying the "operator" $D_{\bar{z}}^{-1}$ to $(2.2)$ and substituting $U=D_{\bar{z}}^{-1} \circ D_{z}$ yields

$$
(I d-U \circ \nu) \dot{f}=(U \circ \dot{\nu}) f .
$$

This equation implies (2.2). The operator $I d-U \circ \nu$ in the left-hand side of this equation is invertible in $L_{2}\left(\mathbb{T}^{3}\right)$ for any $t$ and the norm of the inverse operator is bounded uniformly in $t$, since $U$ is unitary and the module $|\nu|$ is less than 1 and bounded away from 1. Thus, the last equation can be rewritten as

$$
\dot{f}=(I d-U \circ \nu)^{-1}(U \circ \dot{\nu}) f,
$$

which is an ordinary differential equation in $f \in L_{2}\left(\mathbb{T}^{3}\right)$ with a uniformly $L_{2^{-}}$ bounded operator in the right-hand side. As it is shown below (in Proposition 2.7), the inverse $(I d-U \circ \nu)^{-1}$ is also uniformly bounded in each Hilbert-Sobolev spaces $H^{j}\left(\mathbb{T}^{3}\right)$. Therefore, equation (2.6) written in arbitrary Hilbert-Sobolev space has a unique solution with a given initial condition, in particular, with $f(x, 0) \equiv 1$ (the theorem on existence and uniqueness of solution of ordinary differential equation in Banach space with the right-hand side having uniformly bounded derivative $[\mathrm{Ch}]$ ). This solution does not vanish identically on $\mathbb{T}^{3}$ (uniqueness of solution) and belongs to all the spaces $H^{j}\left(\mathbb{T}^{3}\right)$ for each value of $t$. Therefore it is $C^{\infty}\left(\mathbb{T}^{3}\right)$ for any $t$ by Sobolev embedding theorem (see [Ch], p.411). If $\nu$ is analytic in $t$, then so is the right-hand side of (2.6), and hence, the solution is also. Thus, Lemma 2.2 is implied by the following

2.7. Proposition. Let $x=\left(x_{1}, x_{2}, x_{3}\right)$ be affine coordinates on $\mathbb{R}^{3}, \mathbb{T}^{3}=\mathbb{R}^{3} /$ $2 \pi \mathbb{Z}^{3}$. Let $s \geq 0, s \in \mathbb{Z}, U$ be a linear operator in the space of $C^{\infty}$ functions on $\mathbb{T}^{3}$ that commutes with the operators $\frac{\partial}{\partial x_{i}}, i=1,2,3$, and extends to any Sobolev space $H^{j}=H^{j}\left(\mathbb{T}^{3}\right), 0 \leq j \leq s$, up to a unitary operator. Let $0<\delta<1, \nu \in C^{s}\left(\mathbb{T}^{3}\right)$ be a complex-valued function, $|\nu| \leq \delta$. The operator $I d-U \circ \nu$ is invertible and the inverse operator is bounded in all the spaces $H^{j}, 0 \leq j \leq s$. For any $0<\delta<1$, $j \leq s$, there exists a constant $C>0$ (depending only on $\delta$ and $s$ ) such that for any 
complex-valued function $\nu \in C^{s}\left(\mathbb{T}^{3}\right)$ with $|\nu| \leq \delta$

$$
\left\|(I d-U \circ \nu)^{-1}\right\|_{H^{j}} \leq C\left(1+\sum_{k \leq j} \max \left|\frac{\partial^{k} \nu}{\partial x_{i_{1}}, \ldots, \partial x_{i_{k}}}\right|^{j}\right) .
$$

Proof. Let us prove the proposition for $s=1$. For higher $s$ its proof is analogous.

By definition,

$$
\|U \circ \nu\|_{L_{2}} \leq \delta<1
$$

Hence, the operator $I d-U \circ \nu$ is invertible in $L_{2}=H^{0}$ and

$$
(I d-U \circ \nu)^{-1}=I d+\sum_{k=1}^{\infty}(U \circ \nu)^{k}:
$$

the sum of the $L_{2}$ operator norms of the sum entries in the right-hand side of (2.8) is finite by (2.7). Let us show that the operator in the right-hand side of $(2.8)$ is well-defined and bounded in $H^{1}$. To do this, it suffices to show that the sum of the operator $H^{1}$-norms of the same entries is finite.

Let $f \in H^{1}\left(\mathbb{T}^{3}\right)$. Let us estimate the $H^{1}$-norm of the images $(U \circ \nu)^{k} f$. We show that for any $k \in \mathbb{N}$

$$
\left\|\frac{\partial}{\partial x_{r}}\left((U \circ \nu)^{k} f\right)\right\|_{L_{2}}<c k \delta^{k-1}\|f\|_{H^{1}}, \quad c=\delta+\max \left|\frac{\partial \nu}{\partial x_{r}}\right|, \quad r=1,2,3 .
$$

This will imply the finiteness of the operator $H^{1}$-norm of the sum in the righthand side of (2.8) and Proposition 2.7 (with $C=4 \sum_{k \in \mathbb{N}} k \delta^{k-1}=\frac{4}{(1-\delta)^{2}}$ ).

Let us prove (2.9), e.g., for $r=1$. The corresponding derivative in the left-hand side of (2.9) is equal to

$$
(U \circ \nu)^{k} \frac{\partial f}{\partial x_{1}}+\sum_{i=1}^{k}(U \circ \nu)^{k-i} \circ\left(U \circ \frac{\partial \nu}{\partial x_{1}}\right) \circ(U \circ \nu)^{i-1} f
$$

(since $U$ commutes with the partial differentiation by the condition of Proposition 2.7). The $L_{2}$-norm of the first term in the previous formula is no greater than $\delta^{k}\|f\|_{H^{1}}$ by (2.7). Each term in its sum has $L_{2}$-norm no greater than $\delta^{k-1} \max \left|\frac{\partial \nu}{\partial x_{1}}\right|\|f\|_{L_{2}}$ by (2.7). This proves (2.9). The proposition is proved. Lemma 2.2 is proved together with the $C^{\infty}$ versions of Theorems 1.10 and 1.12 .

2.8. Remark. The solution of equation (2.6) with the initial condition $\left.f\right|_{t=0} \equiv 1$ admits the following formula:

$$
f(x, t)=(I d-U \circ \nu)^{-1}(1)=1+U(\nu)+(U \circ \nu \circ U)(\dot{\nu})+\ldots
$$

Indeed, the right-hand side of (2.10) is a well defined $C^{\infty}$ (analytic, dependently on the regularity of $\nu$ in + ) family of $C^{\infty}$ functions on $\mathbb{T}^{3}$, which follows from the uniform boundedness of the operators $(I d-U \circ \nu)^{-1}$ in any given Hilbert-Sobolev 
space. By definition, it satisfies the unit initial condition. Differentiating (2.10) in $t$ yields

$$
(I d-U \circ \dot{\nu})^{-1} \circ(U \circ \nu) \circ(I d-U \circ \nu)^{-1}(1)=(I d-U \circ \nu)^{-1} \circ(U \circ \dot{\nu}) f(x, t) .
$$

Hence, the function (2.10) satisfies (2.6).

Proof of the analytic dependence of the function $f$ on $\mu$. Let $f(x, 1)$ be the solution of the differential equation (2.6) with $\nu=t \mu$. Its analyticity in $\mu$ follows from analyticity of the equation. This proves the last statement of Theorem 1.12.

\subsection{Measurable and analytic cases of Theorem 1.12}

In the case, when the metric $g$ is measurable, the proof of Theorem 1.12 remains valid with obvious changes (e.g. all the differential equations are understood in the sense of distributions) except for the proof of the statement that the constructed $\sigma$ holomorphic differential $f \omega_{\mu}$ is uniformizing. This statement means the following. Take the universal covering of any leaf $L$ equipped with the complex structure induced by $\sigma$. The universal covering (denote it by $\widetilde{L}$ ) is conformally equivalent to $\mathbb{C}$. Then for a generic leaf $L$ with respect to the transversal Lebesgue measure there exists a quasiconformal homeomorphism $\widetilde{L} \rightarrow \mathbb{C}$ whose derivative is equal to the pullback of $f \omega_{\mu}$ in the sense of distributions (this homeomorphism transforms the chosen complex structure on $\widetilde{L}$ to the standard one). Let us prove this statement.

Let $\omega_{\mu}=d z+\mu d \bar{z}$ be the 1 -form $\mathbb{C}$-linear with respect to $\sigma, \nu=t \mu, t \in[0,1]$. Denote by $f(x, t)$ the solution of ordinary differential equation (2.6) in $L_{2}\left(\mathbb{T}^{3}\right)$ with $f(x, 0) \equiv 1$ and put $f=f(x)=f(x, 1)$. The form $f \omega_{\mu}$ is closed (and hence, $\sigma$-holomorphic) on the leaves (in the sense of distributions on the torus) by construction. Let us show that its lifting to the universal covering $\widetilde{L}$ of a generic leaf $L$ (with respect to the Lebesgue transversal measure) is the derivative of a quasiconformal homeomorphism $\widetilde{L} \rightarrow \mathbb{C}$. (The lifting to $\widetilde{L}$ of a function $f$ (a form $\omega_{\mu}$ ) on $L$ will be denoted by the same symbol $f\left(\omega_{\mu}\right)$.) To do this, let us approximate the function $\mu$ by functions $\mu_{k} \in C^{\infty}\left(\mathbb{T}^{3}\right)$ with moduli less than 1 and uniformly bounded away from 1: $\left|\mu_{k}\right|<\delta<1, \mu_{k} \rightarrow \mu$ almost everywhere, as $k \rightarrow \infty$. By $(2.6)_{k}$ denote the equation (2.6) with $\nu=t \mu_{k}$. Let $f_{k}(x, t)$ be its solution with the initial condition $f_{k}(x, 0) \equiv 1$. Put $f_{k}(x)=f_{k}(x, 1)$. Then $f_{k} \rightarrow f$ in $L_{2}\left(\mathbb{T}^{3}\right)$. Indeed, the right-hand sides of $(2.6)_{k}$ depend continuously on $t$, $f$ and the functional parameter $\mu_{k} \in L_{2},\left|\mu_{k}\right| \leq \delta$ (though in general, the operators in the right-hand sides do not converge in the operator norm, as $k \rightarrow \infty$ ). The right-hand sides are uniformly Lipschitz in $f$. This together with the theorem on dependence of solution of ordinary differential equation in Banach space on the parameter [Ch] implies that $f_{k} \rightarrow f$. Let us fix a generic leaf $L$ so that the set $f \neq 0$ has a positive measure in $\widetilde{L}$ and $f_{k} \rightarrow f$ in $L_{2}$ in compact subsets of $\widetilde{L}$. Let us fix a point $y_{0} \in \widetilde{L}$. The form $f_{k} \omega_{\mu_{k}}$ is a uniformizing differential on $\widetilde{L}$ with respect to 
the complex structure defined by $\mu_{k}$, hence, it is the derivative of a quasiconformal diffeomorphism $\psi_{k}: \widetilde{L} \rightarrow \mathbb{C}$ (Theorem 1.1). Let us fix and normalize the latter so that $\psi_{k}\left(y_{0}\right)=0$. By construction, the derivatives $f_{k} \omega_{\mu_{k}}$ of the diffeomorphisms $\psi_{k}$ converge to $f \omega_{\mu}$ in the sense of distributions on $\widetilde{L}$. Let us show that the sequence $\psi_{k}$ converges uniformly in compact sets to a quasiconformal homeomorphism. This homeomorphism will be the one we are looking for: its derivative will be equal to $f \omega_{\mu}$, as the limit of the derivatives $f_{k} \omega_{\mu_{k}}$. To do this, let us fix another point $y_{1} \in \widetilde{L}, y_{1} \neq y_{0}$. Consider the sequence $b_{k}=\left(\psi_{k}\left(y_{1}\right)\right)^{-1}$ and denote $\widetilde{\psi}_{k}=b_{k} \psi_{k}$. The maps $\widetilde{\psi}_{k}$ are quasiconformal diffeomorphisms; each of them transforms the complex structure on $\widetilde{L}$ defined by the form $\omega_{\mu_{k}}$ to the standard one. By definition, they map the points $y_{0}$ and $y_{1}$ to 0 and 1 respectively. They converge uniformly in compact subsets to a quasiconformal homeomorphism that transforms the complex structure defined by the form $\omega_{\mu}$ to the standard one (by theorem on continuous dependence of the normalized uniformizing quasiconformal homeomorphism on the parameter of complex structure $[\mathrm{AhB}])$. Therefore their derivatives also converge (in the sense of distributions) to a nonzero limit. Hence the sequence $b_{k}$ also converges to a nonzero limit, since the contrary would contradict the convergence of the derivatives $f_{k} \omega_{\mu_{k}}$ of the diffeomorphisms $\psi_{k}$. Therefore the initial sequence $\psi_{k}$ also converges uniformly in compact sets to a quasiconformal homeomorphism with the derivative $f \omega_{\mu}$. This proves the measurable version of Theorem 1.12.

Now let us prove Theorem 1.12 in the analytic case using its $C^{\infty}$ version already proved. To do this, it suffices to show that if the function $\mu$ defining the complex structure is analytic, then the corresponding function $f$ defining the uniformizing differential $f \omega_{\mu}$ is also analytic. In this case the function $\mu$ extends holomorphically to some annulus $A_{r}=\left\{x \in \mathbb{C}^{3},|\operatorname{Im} x| \leq r\right\} / 2 \pi \mathbb{Z}$ in complex domain containing the initial torus $\mathbb{T}^{3}$ in the interior (here the complex structure on $A_{r}$ is standard). Let us choose this annulus $A_{r}$ so that $\sup |\mu|_{A_{r}}<1$. Let us show that the corresponding function $f$ extends holomorphically to $A_{r}$. This will prove the analytic version of Theorem 1.12.

The annulus $A_{r}$ is fibered by the tori $\operatorname{Im} x=$ const. The foliation $F$ extends up to a foliation on $A_{r}$ by translating $F$ to the toric fibers $\operatorname{Im} x=$ const.

Let $f(x, t): A_{r} \times[0,1] \rightarrow \mathbb{C}$ be the solution of (2.6) with unit initial condition $f(x, 0) \equiv 1$ : we solve $(2.6)$ on each toric fiber separately. The existence of a solution on each fiber follows from the assumption sup $|\mu|_{A_{r}}<1$. The solutions on the fibers thus obtained depend smoothly on the parameter transversal to the fibers, more precisely, $f(x, t) \in C^{\infty}\left(A_{r}\right)$. This follows from the smooth dependence of the right-hand side of (2.6) on the transversal parameter (more precisely, the smoothness of the family of restrictions to the fibers of the function $\nu=t \mu$ ).

It suffices to show that for any $t \in[0,1]$ the function $f(x, t)$ is holomorphic on $A_{r}$. Indeed, equation (2.6) in a function on $A_{r}$ is an ordinary differential equation preserving the space of holomorphic functions. To prove this, it suffices to show that the operator $(I d-U \circ \nu)^{-1} \circ U \circ \mu$ in its right-hand side (acting on functions on $A_{r}$ and taken along the toric fibers) preserves the space of holomorphic functions. 
This holds for the operator $U$, since it commutes with the antiholomorphic differentiations by construction, and for the operator of multiplication by $\mu$, which is a holomorphic function. The same statement for the operator $(I d-U \circ \nu)^{-1}$, which is given by formula (2.8), follows from the previous statement, which implies that each term of the series in (2.8) preserves the space of holomorphic functions. The analytic versions of Theorems 1.10 and 1.12 are proved.

\subsection{A proof of the integrability of a $C^{\infty}$ complex structure on $\mathbb{T}^{2}$}

In the present subsection we give a proof of Theorem 2.1 stated at the beginning of the section. Its proof repeats that of Theorem 1.12 with small changes.

2.9. Lemma. Let $\nu(x, t): \mathbb{T}^{2} \times[0,1] \rightarrow \mathbb{C}$ be a $C^{\infty}$ family of $C^{\infty}$ functions on $\mathbb{T}^{2},|\nu|<1, \nu(x, 0) \equiv 0, z$ be the complex coordinate on $\mathbb{T}^{2}$. There exists a $C^{\infty}$ family $f(x, t): \mathbb{T}^{2} \times[0,1] \rightarrow \mathbb{C}$ of $C^{\infty}$ functions on $\mathbb{T}^{2}$ that vanish identically in $x \in \mathbb{T}^{2}$ for no parameter value $t$ such that the 1 -form $f \omega_{\nu}=f(d z+\nu d \bar{z})$ on $\mathbb{T}^{2}$ is closed for each parameter value $t$.

The lemma (dealing with a "linear foliation by a single leaf") is a particular case of Lemma 2.2 (when the leaves are tori). Its direct proof repeats that of Lemma 2.2 with obvious changes. We solve equation (2.6) with $U=\left(\frac{\partial}{\partial \bar{z}}\right)^{-1} \circ \frac{\partial}{\partial z}$. This is a unitary operator in $L_{2}\left(\mathbb{T}^{2}\right)$ and Sobolev spaces, which preserves averages and has the eigenbase $1, e^{i\left(n_{1} \operatorname{Re} z+n_{2} \operatorname{Im} z\right)}, n_{1}, n_{2} \in \mathbb{Z}$, with the eigenvalues equal to $\frac{n_{1}-i n_{2}}{n_{1}+i n_{2}}$ for $\left(n_{1}, n_{2}\right) \neq 0$.

Let $\nu=t \mu, f(x, t)$ be the corresponding family in the previous lemma, $f(x)=$ $f(x, 1)$. Then the form $f(x) \omega_{\mu}$ is closed. The function $f$ does not vanish, which follows from Proposition 2.4, as in the proof of Lemma 2.2. The function $f(x)$ depends analytically on $\mu$ by analytic dependence on parameter of a solution of ordinary differential equation in Banach space (here $L_{2}\left(\mathbb{T}^{2}\right)$ ). This remains valid, if we renormalize $f$ to have unit average along a fixed noncontractible closed curve. This proves Theorem 2.1 modulo Proposition 2.4.

\subsection{Zero of holomorphic differential. Proof of Proposition 2.4}

Let us prove the existence of local holomorphic coordinate. Without loss of generality we assume that $\mu(0)=0$ (applying a linear change of variables). One can achieve also that $\mu$ is arbitrarily small with derivatives of orders up to 3 applying a homothety and taking the restriction to a smaller disc centered at 0 . We consider that the disc where $\mu$ is defined is embedded into $\mathbb{T}^{2}$ and extend the function $\mu$ smoothly to $\mathbb{T}^{2}$. We assume that the extended function satisfies the inequality $\|\mu\|_{C^{3}\left(\mathbb{T}^{2}\right)}<\delta$; one can make $\delta$ arbitrarily small. 
Let $\nu(x, t)=t \mu, f(x, t)$ be the corresponding function family from Lemma 2.9 constructed as the solution of differential equation (2.6) with unit initial condition, $f(x)=f(x, 1)$. We show in the next paragraph that $f(0) \neq 0$. Then the local coordinate we are looking for is the function

$$
w(z)=\int_{0}^{z} f(d z+\mu d \bar{z})
$$

Indeed, it is well-defined and holomorphic by definition. Its local univalence follows from the nondegeneracy of its differential $f(0)(d z+\mu d \bar{z})$ at 0 , which is implied by the inequalities $|\mu|<1, f(0) \neq 0$.

Recall that by $(2.10)$,

$$
f(x, t)=(I d-t U \circ \mu)^{-1}(1), \text { where } U=\left(\frac{\partial}{\partial \bar{z}}\right)^{-1} \frac{\partial}{\partial z} .
$$

The functions $f(x, t)$ are equal to 1 , if $\mu \equiv 0$. Let us show that they are close to 1 (and hence, $f(0,1) \neq 0$ ), whenever $\mu$ is small enough with derivatives up to order 3. The operator $(I d-t U \circ \mu)^{-1}$ depends continuously on small $\mu \in C^{3}\left(\mathbb{T}^{2}\right)$ in the $H^{3}\left(\mathbb{T}^{2}\right)$ operator norm. This follows from definition and its uniform boundedness in the same norm (Proposition 2.7, which remains valid in our case). Therefore, if $\|\mu\|_{C^{3}}$ is small enough, then all the functions $f(x, t)$ are close to 1 in $H^{3}$ (and hence, in $C^{0}$, by Sobolev embedding theorem). Proposition 2.4 is proved.

\section{Diophantine foliations. Proofs of Theorems 1.20 and 1.21}

We present only the proof of the three-dimensional $C^{\infty}$ version of Theorem 1.20. This proof remains valid in higher dimensions with obvious changes. The proof of Theorem 1.21 is analogous with some modifications specified at the end of the section.

Let $F$ be a Diophantine foliation whose lifting to the space is the foliation by level planes of a linear function $l(x)=a_{1} x_{1}+a_{2} x_{2}-x_{3}, z=x_{1}+i x_{2}$. Let $\sigma$ be a $C^{\infty}\left(\mathbb{T}^{3}\right)$ family of almost complex structures on the leaves of $F$ (e.g., defined by a smooth metric on $\mathbb{T}^{3}$ ). Let $\omega$ be the corresponding differential form from Theorem 1.12 (whose restriction to each leaf is a uniformizing differential). It is uniquely defined modulo $d l$ up to multiplication by constant, since the leaves of the foliation $F$ are dense ( $F$ is Diophantine). Everyone of the two first equivalent statements of Theorem 1.20 is equivalent to the possibility to choose the form $\omega$ to be closed not only on the leaves, but on the whole torus. Indeed the second statement of Theorem 1.20 implies the existence of a differential 1-form closed on $\mathbb{T}^{3}$ and holomorphic on the leaves. Conversely, for a given closed fiberwise holomorphic 1 -form $\omega$ the Euclidean metric $\omega \bar{\omega}+d l d \bar{l}$ on $\mathbb{T}^{3}$ satisfies (1.7). Thus, Theorem 1.20 is implied by the following 
3.1. Lemma. Let $F, l, \sigma$ be as above, $\mu: \mathbb{T}^{3} \rightarrow \mathbb{C}$ be the $\left(C^{\infty}\right)$ function defining the complex structure family $\sigma$. Let $f: \mathbb{T}^{3} \rightarrow \mathbb{C}$ be a $C^{\infty}$ function such that the 1 -form $\omega=f(x)(d z+\mu d \bar{z})$ be closed along the leaves. There exists a $C^{\infty}$ function $h: \mathbb{T}^{3} \rightarrow \mathbb{C}$ such that the form

$$
f(d z+\mu d \bar{z})-h d l
$$

is closed on $\mathbb{T}^{3}$.

In the proof of the lemma we use the following equivalent reformulation of the condition of closedness of the form (3.1).

3.2. Remark. Let $F, l$ be as above, $f, \mu$ be functions on $\mathbb{T}^{3}$ such that the form $f(d z+\mu d \bar{z})$ be closed along the leaves of $F$. Then a 1 -form (3.1) is closed on $\mathbb{T}^{3}$, if and only if $f, \mu$ and $h$ satisfy the following system of differential equations:

$$
\left\{\begin{array}{l}
\frac{\partial f}{\partial x_{3}}=D_{z} h \\
\frac{\partial(\mu f)}{\partial x_{3}}=D_{\bar{z}} h
\end{array} .\right.
$$

Indeed, let us write down the closedness condition on the form (3.1) in the coordinates $\left(x_{1}, x_{2}, l\right)$. In the new coordinates $\frac{\partial}{\partial z}=D_{z}, \frac{\partial}{\partial \bar{z}}=D_{\bar{z}}$, the operator $\frac{\partial}{\partial l}$ coincides with the operator $-\frac{\partial}{\partial x_{3}}$ corresponding to the coordinates $\left(x_{1}, x_{2}, x_{3}\right)$. By definition, (3.1) is closed on the leaves (or equivalently, $f$ and $\mu$ satisfy (1.4)). Under this assumption, the condition that (3.1) is closed on $\mathbb{T}^{3}$ is equivalent to the system of differential equations $\frac{\partial f}{\partial l}=-\frac{\partial h}{\partial z}, \frac{\partial(\mu f)}{\partial l}=-\frac{\partial h}{\partial \bar{z}}$. Rewriting these equations in the coordinates $\left(x_{1}, x_{2}, x_{3}\right)$ yields $(3.2)$.

Let us show the existence of a $C^{\infty}$ solution $h$ to (3.2). This together with the previous remark will prove Lemma 3.1. To do this, we will use the following characterization of $C^{\infty}$ functions on the torus.

3.3. Remark. A function $h \in L_{1}\left(\mathbb{T}^{3}\right)$ with the Fourier series $\sum_{N} h_{N} e^{i(N, x)}$ is $C^{\infty}$, if and only if

$$
\sum_{N}|N|^{s}\left|h_{N}\right|<\infty \text { for any } s \in \mathbb{N} \text {. }
$$

We use the following equivalent reformulation of the Diophantine condition.

3.4. Remark. Let $F$ be a linear foliation on $\mathbb{T}^{3}, D_{z}$ be the corresponding differential operator from (1.3), $\lambda_{N}$ be its eigenvalues (see Remark 2.5). The foliation $F$ is Diophantine, if and only if there exist $c>0, s \geq 1$ such that for any $N \in \mathbb{Z}^{3} \backslash 0$

$$
\left|\lambda_{N}^{-1}\right| \leq c|N|^{s}
$$


This follows from definition and formula (2.5) for $\lambda_{N}$. In the case, when $F$ is Diophantine, let $C$ be the constant from inequality (1.5). Then one can put $c=\frac{4}{C}$.

We are looking for a solution of the system of equations (3.2). The left-hand side of the first equation is $\frac{\partial f}{\partial x_{3}}$, which has zero average. Define the inverse operator $D_{z}^{-1}$ in the space of infinitely-smooth functions on $\mathbb{T}^{3}$ with zero average (this space is contained in the Hilbert subspace of $L_{2}$ generated by the eigenfunctions $e^{i(N, x)}$ of the operator $D_{z}$ with $\left.N \neq 0\right)$ to have the same eigenfunctions $e^{i(N, x)}$ with the eigenvalues inverse to those of $D_{z}$. This operator is well-defined at least on the eigenfunctions, since the corresponding eigenvalues $\lambda_{N}$ of the operator $D_{z}$ do not vanish (by (3.4)). It is well-defined on the space of infinitely-smooth functions on $\mathbb{T}^{3}$ with zero average by Remark 3.3 and (3.4) and has zero kernel. The function $h=D_{z}^{-1}\left(\frac{\partial f}{\partial x_{3}}\right)$ is the one we are looking for. Indeed it is a $C^{\infty}$ function satisfying the first equation in (3.2). It satisfies the second equation as well. Indeed, applying the operator $D_{z}$ to the second equation and substituting $h=D_{z}^{-1}\left(\frac{\partial f}{\partial x_{3}}\right)$ yields the derivative in $x_{3}$ of equation (1.4), which is satisfied by the assumption that the form $f \omega_{\mu}$ is closed on the leaves. Therefore, the $D_{z}$ images of both parts of the second equation coincide. Hence, these parts coincide themselves: they have zero average, and hence they are obtained from their (coinciding) $D_{z}$ images by applying the operator $D_{z}^{-1}$. This proves Lemma 3.1 and the first statement of Theorem 1.20.

Now let us prove the last statement of Theorem 1.20. Let $F$ be a linear nondiophantine foliation on $\mathbb{T}^{3}$. It suffices to prove the existence of a $C^{\infty}\left(\mathbb{T}^{3}\right)$ family of almost complex structures on the leaves of the foliation $F$ such that there is no $C^{2}$ Euclidean metric on $\mathbb{T}^{3}$ satisfying (1.7). In the case, when the leaves are not dense (i.e., they are tori), for a general $C^{\infty}\left(\mathbb{T}^{3}\right)$ family of complex structures the leaves do not have the same conformal type. This is the case, e.g., if the foliation by tori is given by the planes $x_{3}=$ const, and the function $\mu=\mu\left(x_{3}\right)$ defining the family of complex structures is constant on each leaf but is not constant globally. Then $\omega_{\mu}$ is itself closed on each leaf and is a uniformizing differential. The ratio $\frac{1+\mu}{i(1-\mu)}$ of its periods (taken modulo action of the group $P S L_{2}(\mathbb{R})$ ) is an invariant of complex structure, so, generally, two distinct leaves carry distinct complex structures. The family of complex structures distinct on some pairs of toric leaves is the one we are looking for. Indeed there is no diffeomorphism satisfying the second one of the equivalent statements in Theorem 1.20, since otherwise all the leaves would be conformally equivalent to each other.

Everywhere below we consider that the foliation $F$ has dense leaves. We consider that its lifting to the 3 -space is transversal to the $x_{3}$-axis. For the proof of the last statement of Theorem 1.20 we show the existence of complex-valued functions $\nu, f \in C^{\infty}\left(\mathbb{T}^{3}\right),|\nu|<1, f \neq 0$, such that the form $f \omega_{\nu}$ is closed on the leaves (i.e., $f$ and $\nu$ satisfy (2.1)) and that there is no $C^{2}$ (and even $L_{2}$ ) complex-valued function $h$ on $\mathbb{T}^{3}$ such that the corresponding form (3.1) with $\mu=\nu$ is closed on $\mathbb{T}^{3}$ (or equivalently, (3.2) holds, see Remark 3.2). Then $f \omega_{\nu}$ is a uniformizing 
differential on each leaf with respect to the complex structure defined by $\nu$. The previous statement together with the uniqueness of a uniformizing differential and density of leaves will prove Theorem 1.20.

Let $\lambda_{N}$ be the eigenvalues $(2.5)$ of the operator $D_{z}$. To construct a pair $(\nu, f)$ as in the previous paragraph, let us choose and fix a sequence of distinct multiindices $N^{j}=\left(N_{1}^{j}, N_{2}^{j}, N_{3}^{j}\right) \in \mathbb{Z}^{3}$ such that $\left|\lambda_{N^{j}}\right|<\frac{1}{2}$ and for any $s \in \mathbb{N}$ there exists a number $J \in \mathbb{N}$ such that for any $j>J$

$$
\left|\lambda_{N^{j}}^{-1}\right|>\left|N^{j}\right|^{s}
$$

The existence of such a sequence $N^{j}$ follows from nondiophantine nature of the foliation $F$ (which is contrary to (3.4)). Then $N_{3}^{j} \neq 0$. This follows from the assumption that $\left|\lambda_{N^{j}}\right|<\frac{1}{2}$ and the following inequality: if $N=\left(N_{1}, N_{2}, N_{3}\right) \in$ $\mathbb{Z}^{3} \backslash 0$ is such that $\left|\lambda_{N}\right|<\frac{1}{2}$, then $N_{3} \neq 0$. This inequality follows from (2.5).

Consider the family $f(x, t)=1+t \sum_{j=1}^{\infty} \frac{\lambda_{N^{j}}}{N_{3}^{j}} e^{i\left(N^{j}, x\right)}$ of functions on $\mathbb{T}^{3}$ depending on real parameter $t$. This is a $C^{\infty}$ family of $C^{\infty}$ functions, since the coefficients of the Fourier series in its formula satisfy (3.3) (by (3.5)). Any function $f=f(x, t)$ corresponding to small enough nonzero value of $t$ is a one we are looking for. Indeed, for any fixed nonzero value of the parameter $t$ and any complex-valued function $\mu=\nu$ there is no function $h \in L_{2}\left(\mathbb{T}^{3}\right)$ such that the corresponding form (3.1) is closed, since there is no $h \in L_{2}\left(\mathbb{T}^{3}\right)$ satisfying the first equation in (3.2). Indeed, if an $L_{2}$ function $h$ satisfies the first equation in (3.2), then its Fourier coefficients with the indices $N^{j}$ would be equal to it, which is impossible if $t \neq 0$, since then the $L_{2}$ norm of $h$ would be infinite.

Let us now prove the statement on the existence of a function $\nu$ satisfying (2.1) with the given previous $f$ (Lemma 3.5 below). This will imply Theorem 1.20.

3.5. Lemma. Let $F$ be a linear foliation on $\mathbb{T}^{3}, f(x, t) \neq 0$ be a $C^{\infty}$ family of complex-valued nonvanishing $C^{\infty}$ functions on $\mathbb{T}^{3}$ uniformly depending on real parameter $t, f(x, 0) \equiv 1$. There exists a $C^{\infty}$ family $\nu(x, t)$ of complex-valued $C^{\infty}$ functions on $\mathbb{T}^{3}$ depending on the same parameter $t, \nu(x, 0) \equiv 0$, and satisfying (2.1).

Proof. The lemma is proved by homotopy method analogously to Lemma 2.2 . Differentiating equation (2.1) in $t$ yields (2.2). The solution $\nu(x, t)$ of $(2.2)$ with zero initial condition will be a solution of (2.1). Let $U$ be the operator from Corollary 2.6. Applying subsequently the "operator" $D_{z}^{-1}$ and the multiplication by $f^{-1}$ to $(2.2)$ and substituting $U^{-1}=D_{z}^{-1} \circ D_{\bar{z}}$ yields

$$
\dot{\nu}=\frac{U^{-1} \dot{f}-\nu \dot{f}}{f} \text {. }
$$

The last equation implies (2.2) and has a unique infinitely-smooth solution $\nu(x, t)$ with any given $C^{\infty}$ initial condition (e.g., $\left.\nu(x, 0) \equiv 0\right)$ : its right-hand side has bounded derivative in $\nu$ in any Hilbert-Sobolev norm on any finite segment $[0, \tau]$ 
in the parameter line (as in the proof of Lemma 2.2). This proves Lemma 3.5 and Theorem 1.20.

The proof of Theorem 1.21 is analogous to that of Theorem 1.20. Instead of Remark 3.3 we use the following characterization of analytic functions on $\mathbb{T}^{3}$ :

$$
\begin{aligned}
& \text { a function } h=\sum_{N} h_{N} e^{i(N, x)} \text { on } \mathbb{T}^{3} \text { is analytic, } \\
& \text { if and only if } \varlimsup_{N \rightarrow \infty}\left|h_{N}\right|^{\frac{1}{|N|}}<1 \text {. }
\end{aligned}
$$

\section{Foliations on $\mathbb{T}^{2} \times \mathbb{R}$. Proof of Theorem 1.23}

\subsection{Scheme of the proof of Theorem 1.23}

The proof of Theorem 1.23 is analogous to that of Theorem 1.12. Let $x_{1}, x_{2}$ be the coordinates on $\mathbb{R}^{2}, z=x_{1}+i x_{2}, \mathbb{T}^{2}=\mathbb{R}^{2} / 2 \pi \mathbb{Z}^{2}$. Let $s$ be the additional affine coordinate on the line $\mathbb{R}$ in the product $\mathbb{T}^{2} \times \mathbb{R}$. Let the family $\sigma$ of complex structures on leaves under consideration be defined by a function $\mu: \mathbb{T}^{2} \times \mathbb{R} \rightarrow \mathbb{C}$, $\sup |\mu|<1, \mu \in C_{b}^{\infty}\left(\mathbb{T}^{2} \times \mathbb{R}\right)(\operatorname{see}(1.1))$.

To construct a $C^{\infty}$ family of uniformizing differentials on the leaves, we show that there exists a function $f: \mathbb{T}^{2} \times \mathbb{R} \rightarrow \mathbb{C} \backslash 0, f \in C_{b}^{\infty}\left(\mathbb{T}^{2} \times \mathbb{R}\right)$, such that

the restriction to each leaf of the form $\omega=f(d z+\mu \overline{d z})$ is closed;

the function $f$ is bounded away from zero.

Then the last restriction (4.1) is a nonvanishing holomorphic differential, and its squared module is a complete metric by (4.2). Hence, it is a uniformizing differential.

To construct a function $f$ satisfying (4.1), (4.2), we use the homotopy method (as in the proof of Theorem 1.12). We find a solution $f(x, t), x \in \mathbb{T}^{2} \times \mathbb{R}, t \in[0,1]$, of equation (2.2):

$$
D_{\bar{z}} \dot{f}=\left(D_{z} \circ \nu\right) \dot{f}+\left(D_{z} \circ \mu\right) f, \nu=t \mu,
$$

with the initial condition $\left.f\right|_{t=0} \equiv 1$ that vanishes nowhere and is uniformly bounded away from zero (see $(4.2))$. Then the function $f(x, 1)$ is the one we are looking for.

To solve (4.3), we construct appropriate linear operator $U: C_{b}^{\infty}\left(\mathbb{T}^{2} \times \mathbb{R}\right) \rightarrow$ $C_{b}^{\infty}\left(\mathbb{T}^{2} \times \mathbb{R}\right)($ Lemma 4.4$)$ such that

$$
D_{\bar{z}} \circ U=D_{z}
$$

and $U$ is bounded in the Banach norm (4.8) defined below (the Banach space that is the completion of $C_{b}^{\infty}\left(\mathbb{T}^{2} \times \mathbb{R}\right)$ by this norm will be denoted by $\left.B_{0,0}\right)$. Then the equation

$$
(I d-U \circ \nu) \dot{f}=(U \circ \mu) f
$$


implies (4.3), as in Subsection 2.1.

We show that if $\|\mu\|_{C^{3}}$ is small enough, then the operator $\mu \circ$ of multiplication by $\mu$ in $C_{b}^{\infty}\left(\mathbb{T}^{2} \times \mathbb{R}\right)$ has $B_{0,0}$-operator norm small enough so that $\|U \circ \mu\|<1$. This implies that for any $t \in[0,1]$ there exists a bounded inverse operator $(I d-U \circ \nu)^{-1}$ in $B_{0,0}$ and its norm is bounded uniformly in $t \in[0,1]$ :

$$
\left\|(I d-U \circ \nu)^{-1}\right\|<c<\infty, c \text { does not depend on } t .
$$

This implies the existence and uniqueness of solution $f(x, t)$ of $(4.5)$ in $B_{0,0}$ with any given initial condition (say, $f(x, 0) \equiv 1$ ). Then we show that for any fixed $t \in[0,1] f(x, t) \in C_{b}^{\infty}\left(\mathbb{T}^{2} \times \mathbb{R}\right)$. To do this, we consider additional family of Banach norms \|\|$_{r, k}$ on $C_{b}^{\infty}\left(\mathbb{T}^{2} \times \mathbb{R}\right)$ (see (4.9) below) such that the intersection of the corresponding completions $B_{r, k}$ of $C_{b}^{\infty}\left(\mathbb{T}^{2} \times \mathbb{R}\right)$ coincides with the latter. We show in Subsection 4.4 that inequality (4.6) remains valid in each norm \|\|$_{r, k}$ (with $c=c_{r, k}$ depending on the choice of norm). This will imply the existence and uniqueness of solution in each Banach space $B_{r, k}$. Therefore, the solution of (4.5) with unit initial condition exists and belongs to all these spaces, and hence, to $C_{b}^{\infty}\left(\mathbb{T}^{2} \times \mathbb{R}\right)$.

Denote $C_{b}^{\infty}(\mathbb{R})$ the space of complex-valued $C^{\infty}$ functions on $\mathbb{R}$ with bounded derivatives of each order. For any $f \in C_{b}^{\infty}(\mathbb{R}), r \in \mathbb{N} \cup 0$, put

$$
\|f\|_{C^{r}}=\sum_{0 \leq i \leq r} \sup _{s \in \mathbb{R}}\left|f^{(i)}(s)\right|(\text { cf. (1.8)). }
$$

4.1. Definition. Let $f \in C_{b}^{\infty}\left(\mathbb{T}^{2} \times \mathbb{R}\right)$,

$$
f(x)=\sum_{n=\left(n_{1}, n_{2}\right) \in \mathbb{Z}^{2}} f_{n}(s) e^{i\left(n_{1} x_{1}+n_{2} x_{2}\right)} .
$$

Put

$$
\begin{gathered}
\|f\|_{0,0}=\sum_{n}\left\|f_{n}\right\|_{C^{0}} \text {, and more generally, } \\
\|f\|_{r, k}=\sum_{n \in \mathbb{Z}^{3}, k_{1}+k_{2}=k, k_{i} \geq 0}\left|n_{1}\right|^{k_{1}}\left|n_{2}\right|^{k_{2}}|| f_{n} \|_{C^{r}} ; k_{i}, r \in \mathbb{N} \cup 0, \text { we put }|0|^{0}=1 .
\end{gathered}
$$

4.2. Proposition. For any $f \in C_{b}^{\infty}\left(\mathbb{T}^{2} \times \mathbb{R}\right)$ the expressions (4.8), (4.9) are finite. Conversely, if for a given function $f$ on $\mathbb{T}^{2} \times \mathbb{R}$ the norms (4.9) are finite for all $r, k$, then $f \in C_{b}^{\infty}\left(\mathbb{T}^{2} \times \mathbb{R}\right)$. For any $f \in C_{b}^{\infty}\left(\mathbb{T}^{2} \times \mathbb{R}\right)$

$$
\|f\|_{0,0} \leq 29\|f\|_{C^{3}}
$$

the operator $f \circ$ of multiplication by $f$ is bounded in the norm \|\|$_{0,0}$, and the corresponding operator norm is equal to the vector norm: $\|f \circ\|_{0,0}=\|f\|_{0,0}$.

Proposition 4.2 is proved in Subsection 4.3. 


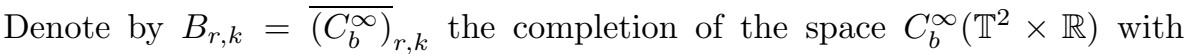
respect to the Banach norm (4.9). By definition, all the spaces $B_{r, k}$ are canonically embedded into the space of bounded continuous functions on $\mathbb{T}^{2} \times \mathbb{R}, B_{r, k}$ is canonically embedded into $B_{r^{\prime}, k^{\prime}}$, if $r \geq r^{\prime}, k \geq k^{\prime}$ and one of these inequalities is strict.

\subsection{Corollary. $\cap_{r, k} B_{r, k}=C_{b}^{\infty}\left(\mathbb{T}^{2} \times \mathbb{R}\right)$.}

As it is shown below, Theorem 1.23 is implied by the two following lemmas.

4.4. Lemma. Let $F$ be a linear foliation on $\mathbb{T}^{2} \times \mathbb{R}, D_{z}, D_{\bar{z}}$ be the corresponding differential operators (1.3). There exists a linear operator $U: C_{b}^{\infty}\left(\mathbb{T}^{2} \times \mathbb{R}\right) \rightarrow$ $C_{b}^{\infty}\left(\mathbb{T}^{2} \times \mathbb{R}\right)$ satisfying $(4.4)$ and commuting with differentiations in $x_{i}, i=1,2,3$, such that

$$
\|U\|_{r, k} \leq 3 \text { for any } r, k \in \mathbb{N} \cup 0
$$

Lemma 4.4 is proved in Subsection 4.2.

4.5. Remark. Let in the conditions of Lemma 4.4 the leaves of $F$ be dense. Then the operator $U$ bounded in the norm \|\|$_{0,0}$ and satisfying the statements of Lemma 4.4 except for (4.11) exists and is unique up to addition of a complexvalued bounded linear functional on $B_{0,0}$ (considered as an operator with values in the space of constant functions). Its uniqueness follows from parabolicity of the leaves of the foliation and Liouville's theorem on bounded entire functions.

4.6. Lemma. Let $F$ be a linear foliation on $\mathbb{T}^{2} \times \mathbb{R}, U$ be the corresponding operator from the previous lemma. Let $\mu \in C_{b}^{\infty}\left(\mathbb{T}^{2} \times \mathbb{R}\right)$ be a complex-valued function such that

$$
\|\mu\|_{C^{3}}<\frac{1}{87}
$$

Then equation (4.5) has a unique $C^{\infty}$ solution $f(x, t)$ with unit initial condition $f(x, 0) \equiv 1$ such that for any fixed $t \in[0,1] f(x, t) \in C_{b}^{\infty}\left(\mathbb{T}^{2} \times \mathbb{R}\right)$. If, more strongly,

$$
\|\mu\|_{C^{3}}<\frac{1}{580}
$$

then $f(x, t)$ vanishes nowhere and is uniformly bounded away from zero.

Lemma 4.6 is proved in Subsection 4.4.

4.7. Remark. Proposition 4.2 and inequalities (4.10)-(4.12) imply that $\|U \circ \mu\|_{0,0}<1$. Inequalities (4.10), (4.11) and (4.13) imply that $\|U \circ \mu\|_{0,0}<\frac{3}{20}$.

Proof of Theorem 1.23. Let $\delta=\frac{1}{580}, \mu \in C_{b}^{\infty}\left(\mathbb{T}^{2} \times \mathbb{R}\right),\|\mu\|_{C^{3}}<\delta$. Thus, inequality 
(4.13) holds, and hence, the solution $f(x, t)$ of (4.5) from Lemma 4.6 does not vanish and is uniformly bounded away from zero. The function $f(x)=f(x, 1)$ satisfies (4.1) and (4.2). This together with the discussion at the beginning of the subsection proves Theorem 1.23. The Addendum to Theorem 1.23 is proved in Subsection 4.5 analogously to the analytic version of Theorem 1.12. The analytic dependence of $f(x)$ on $\mu$ follows from analyticity in $\mu$ of equation (4.5).

\subsection{The operator $U$. Proof of Lemma 4.4}

Let $x_{1}, x_{2}$ be the coordinates on $\mathbb{T}^{2}, s$ be the coordinate on $\mathbb{R}$. Let the linear foliation $F$ under consideration be tangent to the constant vector fields

$$
\frac{\partial}{\partial x_{1}}+a_{1} \frac{\partial}{\partial s}, \quad \frac{\partial}{\partial x_{2}}+a_{2} \frac{\partial}{\partial s} .
$$

Define

$$
a=a_{1}+i a_{2} .
$$

For simplicity everywhere below in the present subsection for a function $f \in$ $C_{b}^{\infty}\left(\mathbb{T}^{2} \times \mathbb{R}\right)$ we will write down its Fourier expansion in $\left(x_{1}, x_{2}\right)$ in the following form:

$$
f(x)=\sum_{n \in \mathbb{Z}+i \mathbb{Z}} f_{n}(s) e^{\frac{\bar{n} z-n \bar{z}}{2}}, \quad z=x_{1}+i x_{2} .
$$

Let us write down formulas for the operators $D_{z}, D_{\bar{z}}$ in terms of the Fourier expansion. To do this, let us introduce the following notations. For any $c \in \mathbb{C}$ consider the following differential operators $L_{a, c}, L_{a, c}^{*}=L_{-\bar{a}, \bar{c}}: C_{b}^{\infty}(\mathbb{R}) \rightarrow C_{b}^{\infty}(\mathbb{R})$ :

$$
L_{a, c} f=\bar{c} f+\bar{a} \frac{d f}{d s}, \quad L_{a, c}^{*} f=c f-a \frac{d f}{d s} .
$$

4.8. Remark. The operators $L_{a, c}$ and $L_{a, c}^{*}=L_{-\bar{a}, \bar{c}}$ are conjugated as those acting on the subspace in $L_{2}(\mathbb{R})$ of functions with first derivatives in the class $L_{2}$.

It follows from definition (see also (2.4)) that for any function (4.15)

$$
2 D_{z} f=\sum_{n \in \mathbb{Z}+i \mathbb{Z}}\left(L_{a, n} f_{n}\right)(s) e^{\frac{\bar{n} z-n \bar{z}}{2}}, 2 D_{\bar{z}} f=-\sum_{n \in \mathbb{Z}+i \mathbb{Z}}\left(L_{a, n}^{*} f_{n}\right)(s) e^{\frac{\bar{n} z-n \bar{z}}{2}} .
$$

To define the operator $U=D_{\bar{z}}^{-1} \circ D_{z}$, we firstly define the operator $\left(L_{a, c}^{*}\right)^{-1} \circ$ $L_{a, c}$ in $C_{b}^{\infty}\left(\mathbb{T}^{2} \times \mathbb{R}\right)$ for any $a, c \in \mathbb{C}$ (not vanishing simultaneously) and then put

$$
U f=-\sum_{n \in \mathbb{Z}+i \mathbb{Z}}\left(\left(L_{a, n}^{*}\right)^{-1} \circ L_{a, n} f_{n}\right)(s) e^{\frac{\bar{n} z-n \bar{z}}{2}} .
$$

Case 1: $a=0$. Then $L_{a, c}=\bar{c}, L_{a, c}^{*}=c$, and we put $\left(L_{a, c}^{*}\right)^{-1} \circ L_{a, c}=\overline{\bar{c}}$. 
Case 2: $a \neq 0, \frac{c}{a} \in i \mathbb{R}$. Then $L_{a, c}=-\frac{\bar{a}}{a} L_{a, c}^{*}$ by (4.16). Put

$$
\left(L_{a, c}^{*}\right)^{-1} \circ L_{a, c}=-\frac{\bar{a}}{a} .
$$

Case 3: $a \neq 0, \frac{c}{a} \notin i \mathbb{R}$. Let us firstly construct the inverse operator $\left(L_{a, c}^{*}\right)^{-1}$.

4.9. Lemma. Let $a, c \in \mathbb{C}, a \neq 0, \frac{c}{a} \notin i \mathbb{R}$. Let $L_{a, c}^{*}: C_{b}^{\infty}(\mathbb{R}) \rightarrow C_{b}^{\infty}(\mathbb{R})$ be the corresponding differential operator defined in (4.16). The operator $L_{a, c}^{*}$ has a unique inverse $\left(L_{a, c}^{*}\right)^{-1}: C_{b}^{\infty}(\mathbb{R}) \rightarrow C_{b}^{\infty}(\mathbb{R})$. More precisely, for any $g \in C_{b}^{\infty}(\mathbb{R})$ there is a unique $f \in C_{b}^{\infty}(\mathbb{R})$ such that $L_{a, c}^{*} f=g$. In addition,

$$
\begin{gathered}
\left\|\left(L_{a, c}^{*}\right)^{-1}\right\|_{C^{0}}=1 \text {, if } a=c=1 ; \\
\left\|\left(L_{a, c}^{*}\right)^{-1}\right\|_{C^{0}}=\left|a \operatorname{Re}\left(\frac{c}{a}\right)\right|^{-1} \text { in the general case. }
\end{gathered}
$$

This norm is equal to the module of the eigenvalue of $\left(L_{a, c}^{*}\right)^{-1}$ at its eigenfunction $g(s)=e^{i \operatorname{Im}\left(\frac{c}{a}\right) s}$.

Proof. It suffices to prove Lemma 4.9 in the case when $\frac{c}{a} \in \mathbb{R}$ : in the general case the transformation $u=e^{-i \operatorname{Im}\left(\frac{c}{a}\right) s} f$ (which is a $C^{0}$-isometry of the space $C_{b}^{\infty}(\mathbb{R})$ ) conjugates $L_{a, c}^{*}$ to $L_{a, c^{\prime}}^{*}$ with $\frac{c^{\prime}}{a}=\operatorname{Re}\left(\frac{c}{a}\right) \in \mathbb{R}$. So, let $\frac{c}{a} \in \mathbb{R}$. Without loss of generality we assume that $a=c=1$ : one can achieve this by applying linear change of the variable and by multiplying the operator $L_{a, c}^{*}$ by $c^{-1}$. (Applying the inverse changes (taking $L_{-1,1}^{*}$ to a given arbitrary $L_{a, c}^{*}$ ) transforms (4.19) in the case $a=c=1$ to its generalization (following (4.19)) for arbitrary $a, c$.) Let us solve the equation

$$
L_{a, c}^{*} f=f-\frac{d f}{d s}=g .
$$

Taking $f$ to the right-hand side yields

$$
\dot{f}=f-g, \dot{f}=\frac{d f}{d s} .
$$

It suffices to show that for any $g \in C_{b}^{\infty}(\mathbb{R})(4.20)$ has a unique solution $f \in C_{b}^{\infty}$ and the latter satisfies the inequality $\sup |f| \leq \sup |g|$.

\section{Uniqueness of bounded solution}

Suppose the contrary: there are two distinct bounded solutions of (4.20). Their difference is a bounded solution of the corresponding linear homogeneous equation (i.e., that with $g=0$ ). Hence, it is equal to $q e^{s}, q \in \mathbb{C} \backslash 0$, which is unbounded a contradiction.

\section{Existence of bounded solution}

4.10. Proposition. Let $s_{0} \in \mathbb{R}, g \in C^{\infty}\left(-\infty, s_{0}\right]$ be a bounded function, $f$ be the solution of equation (4.20) on the semiinterval $\left(-\infty, s_{0}\right]$ with the initial condition 
$f\left(s_{0}\right)=g\left(s_{0}\right)$. Then $f$ is bounded and moreover

$$
|f(y)| \leq \sup _{\left(-\infty, s_{0}\right]}|g| \text { for any } y \in\left(-\infty, s_{0}\right]
$$

Proof. The module $|f|$ is strictly increasing at the points $y<s_{0}$ where

$$
|f(y)|>|g(y)| \text {. }
$$

This follows immediately from (4.20): if (4.22) holds, then the right-hand side of (4.20) will be positive if $f>0$ and negative otherwise. By the initial condition, $f\left(s_{0}\right)=g\left(s_{0}\right)$; thus, inequality (4.21) holds at $s_{0}:\left|f\left(s_{0}\right)\right| \leq \sup |g|$. Suppose the contrary to Proposition 4.10, i.e., (4.21) is wrong at some point $y<s_{0}$ : $|f(y)|>$ $\sup |g|$. Then $|f(y)|>\sup |g| \geq\left|f\left(s_{0}\right)\right|$. On the other hand, $y<s_{0}$. Therefore, one can choose the point $y$ so that $|f|^{\prime}(y)<0$ and the inequality $|f(y)|>\sup |g|$ remains valid (hence, (4.22) holds). This contradicts to the previous increasing property of $|f|$. Proposition 4.10 is proved.

Consider the family $f_{s_{0}}$ of solutions of (4.20) from Proposition 4.10 corresponding to different starting points $s_{0}$ (each solution is restricted to its own semiinterval $\left.\left(-\infty, s_{0}\right]\right)$. This family is uniformly bounded by $(4.21)$ (the functions are bounded on their semiintervals $\left(-\infty, s_{0}\right]$ by one and the same constant). Therefore, by (4.20), their first derivatives are also uniformly bounded in the same sense. Hence, the family $f_{s_{0}}$ is precompact in $C^{r}$ for any $r \in \mathbb{N}$ on each segment of $\mathbb{R}$, as $s_{0} \rightarrow+\infty$. Therefore, it contains a subsequence converging in the $C^{1}$ norm: this subsequence corresponds to a sequence of points $s_{0}$ converging to $+\infty$. A limit of the converging sequence is a bounded solution of (4.20) by definition (denote it by $f)$. It follows from the boundedness and equation (4.20) that each derivative $f^{(k)}$ is bounded, thus, $f \in C_{b}^{\infty}(\mathbb{R})$. The statement of existence is proved. Inequality (4.21), which holds for any $f_{s_{0}}$, implies that $\sup |f| \leq \sup |g|$. This implies that the norm in (4.19) is no greater than 1. If $g=$ const, then $f=g$, which implies (4.19) and the last statement of Lemma 4.9 for the chosen $a=c=1$. Lemma 4.9 is proved.

4.11. Corollary. Let $a, c \in \mathbb{C}, a \neq 0, \frac{c}{a} \notin i \mathbb{R}$. Let $L_{a, c}, L_{a, c}^{*}$ be the operators from (4.16), $\left(L_{a, c}^{*}\right)^{-1}$ be the inverse operator from Lemma 4.9. Then

$$
\left\|\left(L_{a, c}^{*}\right)^{-1} \circ L_{a, c}\right\|_{C^{0}} \leq 3 .
$$

Proof. Without loss of generality, we suppose that $a=c=1$, as in the proof of Lemma 4.9. Then, by definition,

$$
L_{a, c}=-L_{a, c}^{*}+2 .
$$

Therefore,

$$
\left(L_{a, c}^{*}\right)^{-1} \circ L_{a, c}=-1+2\left(L_{a, c}^{*}\right)^{-1}
$$


This together with (4.19) implies the Corollary.

4.12. Remark. The operators $L_{a, c}, L_{a, c}^{*}$ from (4.16) commute with differentiations, hence, so does $\left(L_{a, c}^{*}\right)^{-1} \circ L_{a, c}$.

The last remark together with the previous corollary imply

4.13. Corollary. Let $a, c \in \mathbb{C}, a \neq 0, \frac{c}{a} \notin i \mathbb{R}$. Let $L_{a, c}, L_{a, c}^{*}$ be the operators from (4.16), $\left(L_{a, c}^{*}\right)^{-1}$ be the inverse from Lemma 4.9. Then

$$
\left\|\left(L_{a, c}^{*}\right)^{-1} \circ L_{a, c}\right\|_{C^{r}} \leq 3 \text { for any } r \in \mathbb{N} \cup 0 .
$$

Now all the operators $\left(L_{a, c}^{*}\right)^{-1} \circ L_{a, c}$ are defined. Let $U$ be the corresponding operator (4.18) on functions on $\mathbb{T}^{2} \times \mathbb{R}$. This operator satisfies the statements of Lemma 4.4. Indeed, inequality (4.11) follows from definition and (4.23). Together with Proposition 4.2 it implies that $U$ is a well defined operator acting on the space $C_{b}^{\infty}\left(\mathbb{T}^{2} \times \mathbb{R}\right)$. Equality (4.4) follows from definition. The operator $U$ commutes with differentiations by definition and the previous remark. Lemma 4.4 is proved.

\subsection{Properties of the norms. Proof of Proposition 4.2}

In the proof of Proposition 4.2 we use the following

4.14. Remark. For any $k=\left(k_{1}, k_{2}, 0\right), k_{1}, k_{2} \in \mathbb{N} \cup 0$, and any $f \in C_{b}^{\infty}\left(\mathbb{T}^{2} \times\right.$ $\mathbb{R})$ with Fourier expansion (4.7) the function $i^{|k|} n_{1}^{k_{1}} n_{2}^{k_{2}} f_{n}(s) e^{i\left(n_{1} x_{1}+n_{2} x_{2}\right)}$ is the Fourier $\left(n_{1}, n_{2}\right)$-harmonic in $\left(x_{1}, x_{2}\right)$ of the derivative $\frac{d^{|k|} f}{d x^{k}}$. Therefore,

$$
\left|n_{1}\right|^{k_{1}}\left|n_{2}\right|^{k_{2}}\left|f_{n}\right| \leq \sup \left|\frac{d^{|k|} f}{d x^{k}}\right| \text {. }
$$

Let us firstly prove inequality (4.10) of Proposition 4.2:

$$
\|f\|_{0,0} \leq 29\|f\|_{C^{3}} .
$$

By (4.24), for any $n \neq 0$

$$
\begin{gathered}
\left(\left|n_{1}\right|^{3}+\left|n_{1}^{2} n_{2}\right|+\left|n_{1} n_{2}^{2}\right|+\left|n_{2}\right|^{3}\right)\left|f_{n}\right| \leq\|f\|_{C^{3}}, \text { so } \\
\left|f_{n}\right| \leq \frac{\|f\|_{C^{3}}}{|n|\left(\left|n_{1}\right|^{2}+\left|n_{2}\right|^{2}\right)} \leq \frac{2\|f\|_{C^{3}}}{|n|^{3}},|n|=\left|n_{1}\right|+\left|n_{2}\right|
\end{gathered}
$$

(the quadratic mean inequality). Therefore,

$$
\|f\|_{0,0} \leq\left|f_{0}\right|+2\|f\|_{C^{3}} \sum_{n \neq 0} \frac{1}{|n|^{3}} \leq\|f\|_{C^{3}}\left(1+2 \sum_{n \neq 0} \frac{1}{|n|^{3}}\right) .
$$


Let us estimate the last sum. Each its term is no greater than $\frac{1}{\max \left\{\left|n_{1}\right|^{3},\left|n_{2}\right|^{3}\right\}}$. Therefore, the sum is no greater than

$$
\sum_{m=1}^{\infty} \sum_{\max \left\{\left|n_{1}\right|,\left|n_{2}\right|\right\}=m} m^{-3}=\sum_{m=1}^{\infty} \frac{8 m}{m^{3}}=8 \sum_{m=1}^{\infty} m^{-2}=4 \frac{\pi^{2}}{3}<14:
$$

for a fixed $m \in \mathbb{N}$ the points $n=\left(n_{1}, n_{2}\right)$ such that $\max _{i=1,2}\left|n_{i}\right|=m$ lie in the boundary of the square $[-m, m]^{2}$ and their number is equal to $8 m$. This together with (4.25) implies (4.10) and in particular, finiteness of the $(0,0)$-norm of a function from $C_{b}^{\infty}\left(\mathbb{T}^{2} \times \mathbb{R}\right)$.

Finiteness of any norm \|\|$_{r, k}$ of a function from $C_{b}^{\infty}\left(\mathbb{T}^{2} \times \mathbb{R}\right)$ is proved analogously using (4.24).

Let us prove that conversely, for any function $f$ on $\mathbb{T}^{2} \times \mathbb{R}$ finiteness of all the norms $\|f\|_{r, k}$ implies that $f \in C_{b}^{\infty}\left(\mathbb{T}^{2} \times \mathbb{R}\right)$. Let a function $f$ has all these norms finite. This together with the previous remark imply that any derivative of the Fourier series in $\left(x_{1}, x_{2}\right)$ of $f$ is a uniformly absolutely converging series. Therefore, the corresponding derivative of $f$ exists and is uniformly bounded. Thus, $f \in C_{b}^{\infty}\left(\mathbb{T}^{2} \times \mathbb{R}\right)$.

The statement of Proposition 4.2 that the norm $\|f\|_{0,0}$ coincides with the corresponding norm of the operator of multiplication by $f$ follows from definition: for any $h \in C_{b}^{\infty}\left(\mathbb{T}^{2} \times \mathbb{R}\right)$

$$
\|f h\|_{0,0}=\sum_{j}\left\|\sum_{i} f_{i} h_{j-i}(s)\right\|_{C^{0}} \leq \sum\left\|f_{i}\right\|_{C^{0}} \sum\left\|h_{j}\right\|_{C^{0}}=\|f\|_{0,0}\|h\|_{0,0},
$$

and the equality takes place for $h \equiv 1$. Proposition 4.2 is proved.

\subsection{Smoothness and nonvanishing. Proof of Lemma 4.6}

In the proof of Lemma 4.6 we use the following inequalities valid for any $f \in$ $C_{b}^{\infty}\left(\mathbb{T}^{2} \times \mathbb{R}\right)$ :

$$
\begin{gathered}
\|f\|_{0,0} \geq\|f\|_{C^{0}} \\
\text { for } k>0 \quad\|f\|_{r, k}=\sum_{\gamma=\left(\gamma_{1}, \gamma_{2}, 0\right),|\gamma|=k}\left\|\frac{\partial^{|\gamma|} f}{\partial x^{\gamma}}\right\|_{r, 0}+\left\|f_{0}\right\|_{C^{r}} \\
\leq \sum_{\gamma=\left(\gamma_{1}, \gamma_{2}, 0\right),|\gamma|=k}\left\|\frac{\partial^{|\gamma|} f}{\partial x^{\gamma}}\right\|_{r, 0}+\sum_{\alpha=0}^{r}\left\|\frac{\partial^{\alpha} f}{\partial s^{\alpha}}\right\|_{0,0} .
\end{gathered}
$$

Inequality (4.26) follows from definition. The equality in (4.27) follows from the previous remark and definition. The inequality in (4.27) follows from inequality (4.26) applied to the derivatives of $f$ in $s$ and the statement that the $C^{0}$-norm of a function on $\mathbb{T}^{2} \times \mathbb{R}$ is no less than that of its average over $\mathbb{T}^{2}$.

Let us prove the first statement of Lemma 4.6: if inequality (4.12) holds, i.e., if $\|\mu\|_{C^{3}}<\frac{1}{87}$, then equation (4.5) has a unique $C^{\infty}$ solution $f(x, t)$ with $f(x, 0) \equiv 1$ 
such that $f(x, t) \in C_{b}^{\infty}\left(\mathbb{T}^{2} \times \mathbb{R}\right)$ for any $t \in[0,1]$. To do this, we show that

$$
\left\|(I d-U \circ \nu)^{-1}\right\|_{r, k}<c_{r, k}<\infty, \quad c_{r, k}>0 \text { depends only on } r, k .
$$

This will imply existence and uniqueness of solution $f$ of (4.5) in each Banach space $B_{r, k}$, and hence, in $C_{b}^{\infty}\left(\mathbb{T}^{2} \times \mathbb{R}\right)$ (see the discussion preceding Definition 4.1 in 4.1$)$.

We use the formula

$$
(I d-U \circ \nu)^{-1}=\sum_{p=0}^{\infty} t^{p}(U \circ \mu)^{p} .
$$

Put

$$
\delta=\|U \circ \mu\|_{0,0}, \text { then } \delta<1
$$

by (4.12), Proposition 4.2 (which imply together that $\|\mu\|_{0,0}=\|\mu \circ\|_{0,0}<\frac{1}{3}$ ) and inequality (4.11), which implies that $\|U\|_{0,0} \leq 3$. This together with (4.29) implies (4.28) for $r=k=0$.

Now let us prove (4.28) for $r=0, k=1$. For higher $r, k$ the proof is analogous. Let us estimate $\left\|(U \circ \mu)^{p}\right\|_{0,1}$. Let $f \in C_{b}^{\infty}\left(\mathbb{T}^{2} \times \mathbb{R}\right)$. By (4.27),

$$
\left\|(U \circ \mu)^{p} f\right\|_{0,1} \leq \sum_{i=1,2}\left\|\frac{\partial}{\partial x_{i}}\left((U \circ \mu)^{p} f\right)\right\|_{0,0}+\left\|(U \circ \mu)^{p} f\right\|_{0,0} .
$$

Let us estimate the norm of each derivative in (4.30). We have

$$
\frac{\partial}{\partial x_{i}}\left((U \circ \mu)^{p} f\right)=(U \circ \mu)^{p}\left(\frac{\partial f}{\partial x_{i}}\right)+\sum_{\sigma=0}^{p-1}(U \circ \mu)^{\sigma} \circ U \circ \mu_{x_{i}} \circ(U \circ \mu)^{p-1-\sigma} f
$$

(the operator $U$ commutes with the differentiations). Therefore,

$\left\|\frac{\partial}{\partial x_{i}}\left((U \circ \mu)^{p} f\right)\right\|_{0,0} \leq \delta^{p}\left\|\frac{\partial f}{\partial x_{i}}\right\|_{0,0}+p \delta^{p-1}\|U\|_{0,0}\left\|\frac{\partial \mu}{\partial x_{i}}\right\|_{0,0}\|f\|_{0,0}, \delta=\|U \circ \mu\|_{0,0}$.

Recall that $\|U\|_{0,0} \leq 3$ by (4.11) and $\left\|\frac{\partial \mu}{\partial x_{i}}\right\|_{0,0}<c^{\prime}<+\infty\left(c^{\prime}>0\right.$, by Proposition 4.2 and since $\left.\frac{\partial \mu}{\partial x_{3}} \in C_{b}^{\infty}\left(\mathbb{T}^{2} \times \mathbb{R}\right)\right)$. Therefore, the right-hand side of the previous inequality is no greater than

$\left(\delta^{p}+p \delta^{p-1}\right)\left(1+\|U\|_{0,0}\left\|\frac{\partial \mu}{\partial x_{i}}\right\|_{0,0}\right)\left(\left\|\frac{\partial f}{\partial x_{i}}\right\|_{0,0}+\|f\|_{0,0}\right) \leq\left(1+3 c^{\prime}\right)(p+1) \delta^{p-1}\|f\|_{0,1}$.

Thus, by (4.30) and the two last inequalities,

$\left\|(U \circ \mu)^{p} f\right\|_{0,1} \leq 2\left(1+3 c^{\prime}\right)(p+1) \delta^{p-1}\|f\|_{0,1}+\delta^{p}\|f\|_{0,0} \leq\left(3+6 c^{\prime}\right)(p+1) \delta^{p-1}\|f\|_{0,1}$.

Therefore,

$$
\left\|(U \circ \mu)^{p}\right\|_{0,1} \leq\left(3+6 c^{\prime}\right)(p+1) \delta^{p-1} .
$$

The series in $p$ with the terms from the right-hand side of the last inequality converges, since $\delta<1$. Therefore, so does the series of the left-hand sides, and 
hence, the series of the norms of the sum entries in (4.29). This together with (4.29) implies (4.28) for $r=0, k=1$. The first statement of Lemma 4.6 is proved.

Now let us prove the second statement of Lemma 4.6: if inequality (4.13) holds, i.e., if

$$
\|\mu\|_{C^{3}}<\frac{1}{580}
$$

then the solution $f(x, t)$ of $(4.5)$ with $f(x, 0) \equiv 1$ vanishes nowhere and is uniformly bounded away from zero. To do this, it suffices to show that there exists a $c>0$, $c<1$, such that for any $x \in \mathbb{T}^{2} \times \mathbb{R}, t \in[0,1]|1-f(x, t)|<c<1$.

By (4.26), for the proof of the previous inequality it suffices to show that there exists a $0<c<1$ such that for any $t \in[0,1]$

$$
\|1-f(x, t)\|_{0,0}<c .
$$

Let us prove (4.31). To do this, we use the well-known exponential estimate of the norm of a solution of bounded linear ordinary differential equation (see $[\mathrm{Ar}]$ ). Equation (4.5) can be rewritten as

$$
\dot{f}=B(t) f, B(t)=(I d-U \circ \nu)^{-1} \circ U \circ \mu .
$$

Therefore, for any $t \in[0,1]$

$$
\|f\|_{0,0} \leq \exp \left(\sup _{\tau}\|B(\tau)\|_{0,0}\right) .
$$

Let us estimate $\|B(t)\|$. Recall that $\|\mu\|_{0,0} \leq 29\|\mu\|_{C^{3}}<\frac{1}{20}$ by (4.10) and (4.13), $\|U\|_{0,0} \leq 3$. Therefore,

$$
\begin{gathered}
\left\|(I d-U \circ \nu)^{-1}\right\|_{0,0} \leq \sum_{p=0}^{\infty}\|U\|_{0,0}^{p}\|\mu\|_{0,0}^{p}<\sum_{p=0}^{\infty}\left(\frac{3}{20}\right)^{p}=\frac{1}{1-\frac{3}{20}}, \\
\|B(t)\|_{0,0}<\frac{\|U\|_{0,0}\|\mu\|_{0,0}}{1-\frac{3}{20}} \leq \frac{3}{20\left(1-\frac{3}{20}\right)}<0.3 .
\end{gathered}
$$

This together with (4.33) implies that

$$
\|f\|_{0,0} \leq e^{0.3}
$$

Now let us estimate $\|1-f(x, t)\|_{0,0}$. By (4.32), (4.34) and (4.35),

$$
\|1-f(x, t)\|_{0,0} \leq \int_{0}^{1}\|B(t) f(x, t)\|_{0,0} d t \leq \sup \left(\|B(t)\|_{0,0}\|f\|_{0,0}\right) \leq 0.3 e^{0.3}<1 .
$$

This together with (4.26) proves (4.31). Lemma 4.6 is proved.

\subsection{The analytic case. Proof of the Addendum to Theorem $\mathbf{1 . 2 3}$}

By the conditions of the Addendum, the function $\mu$ extends holomorphically to a complex neighborhood

$$
V_{\Delta}=\left\{\left|\operatorname{Im} x_{1}\right|+\left|\operatorname{Im} x_{2}\right|+|\operatorname{Im} s|<\Delta\right\}, \Delta>0,
$$


of the real space $\mathbb{T}^{2} \times \mathbb{R}$ so that $\|\mu\|_{C^{3}\left(T_{\left.y_{1}, y_{2}, y_{3}\right)}\right)}<\delta=\frac{1}{580}, T_{y_{1}, y_{2}, y_{3}}=\left\{\operatorname{Im} x_{1}=\right.$ $\left.y_{1}, \operatorname{Im} x_{2}=y_{2}, \operatorname{Im} s=y_{3}\right\}$. On each fiber $T_{y_{1}, y_{2}, y_{3}}$ the function $\mu$ is $C_{b}^{\infty}$ : moreover, its derivatives in all the coordinates of $V_{\Delta}$ of each order are bounded on any subset of the type $V_{\Delta^{\prime}} \subset V_{\Delta}, \Delta^{\prime}<\Delta$. Indeed, all its first derivatives are bounded on $V_{\Delta^{\prime}}$ (holomorphy of $|\mu|$, the inequality $|\mu|<1$ and the Schwarz lemma). The boundedness of the second derivatives, etc. is proved analogously.

For the proof of the Addendum we prove the existence of a holomorphic function $f$ on $V_{\Delta}$ such that the form $f \omega_{\mu}$ is a uniformizing differential on the leaves of the foliation $F$ translated to the fibers $T_{y_{1}, y_{2}, y_{3}}$.

On each fiber $T_{y_{1}, y_{2}, y_{3}}$ there exists a smooth $C_{b}^{\infty}$ solution $f(x, t)$ of linear equation (4.5) with unit initial condition that vanishes nowhere and is bounded away from zero (Lemma 4.6). The same linear equation preserves the space of holomorphic functions on $V_{\Delta}$, as at the end of Subsection 2.2. Therefore, $f(x, t)$ is holomorphic on $V_{\Delta}$. The function $f=f(x, 1)$ is a one we are looking for. The Addendum is proved.

\section{Nondifferentiable counterexample on $\mathbb{T}^{3}$. Proof of Theorem 1.25}

\subsection{A counterexample and the scheme of the proof of Theorem $\mathbf{1 . 2 5}$}

Let $S^{1}=\mathbb{R} / 2 \pi \mathbb{Z}, y$ be the affine coordinate on $\mathbb{R}$. Let $v=v(y) \frac{\partial}{\partial y}$ be a vector field either on $S^{1}(v(y)$ is $2 \pi$-periodic), or on the segment [0, $\pi]$. By $F_{v}$ we denote the foliation on the 3 -torus $\mathbb{T}^{3}=\mathbb{T}^{2} \times S^{1}$ (or on the product $\mathbb{T}^{2} \times[0, \pi]$ ) with the coordinates $x=\left(x_{1}, x_{2}, y\right)$ that is tangent to the planes generated by the vector fields

$$
\frac{\partial}{\partial x_{1}}, \frac{\partial}{\partial x_{2}}+v(y) \frac{\partial}{\partial y} .
$$

We show that the foliation $F_{v}$ with

$$
v(y)=3 \sin y
$$

satisfies the statements of Theorem 1.25.

5.1. Remark. Any foliation $F_{v}$ is well-defined and is invariant under translations of $\mathbb{T}^{2}$. In particular, the set $v(y)=0$ is invariant for $F_{v}$ and is foliated by tori. Any other leaf is homeomorphic to cylinder. In particular, in the above case, when $v(y)=3 \sin y$, there are two toric leaves: $y=0, \pi$. Each leaf is fibered by circles tangent to the field $\frac{\partial}{\partial x_{1}}$, and these circles are not contractible as loops in the leaf. The foliation $F_{v}$ corresponding to a constant vector field $v$ is a linear foliation.

Now let us define the family of complex structures on the leaves for which we will prove Theorem 1.25. Let $\chi(y) \in C^{\infty}\left(S^{1}\right)$,

$$
\chi \text { is real, } \chi(0)=0,|\chi|<1 \text {, put } \mu(x)=\chi(y) e^{-i x_{1}} .
$$


Let $\sigma$ be the family of complex structures on the leaves defined by the previous function $\mu$. We show that for appropriate function $\chi$ as in (5.1) (that may be taken analytic) the above foliation $F_{v}$ and the complex structure $\sigma$ satisfy the statements of Theorem 1.25.

It suffices to prove the statement of Theorem 1.25 for the foliation $F_{v}$ restricted to $\mathbb{T}^{2} \times[0, \pi]$. We show (Lemma 5.4 below) that there always exists a unique continuous family of uniformizing metrics and a unique continuous family of uniformizing differentials on the leaves, both up to multiplication by constant. The family of uniformizing differentials has the form $f\left(x_{1}, x_{2}, y\right) \omega_{\mu}, f: \mathbb{T}^{2} \times[0, \pi] \rightarrow \mathbb{C} \backslash 0$; the uniformizing metrics are their squared modules $|f|^{2}\left|\omega_{\mu}\right|^{2}$. Then we prove (as sketched below) that for appropriately chosen function $\chi$ the corresponding function $|f|^{2}$ (and hence, the uniformizing metric) is not differentiable in $y$ at the toric leaf $y=0$.

Let us sketch the proof of the nondifferentiability. The change $y \mapsto s$ of the variable $y$ to the time $s$ of the vector field $v(y) \frac{\partial}{\partial y}$ transforms $\left.F_{v}\right|_{\mathbb{T}^{2} \times[0, \pi]}$ to the linear foliation $F$ on $\mathbb{T}^{2} \times \mathbb{R}$ by cylinders called standard,

$$
F \text { is tangent to the vector fields } \frac{\partial}{\partial x_{1}}, \frac{\partial}{\partial x_{2}}+\frac{\partial}{\partial s},
$$

with the induced family of complex structures on the leaves. The corresponding family of uniformizing differentials is given by the form $f\left(x_{1}, x_{2}, y(s)\right) \omega_{\mu}$. We analyze the uniformizing differentials of the linear foliation by cylinders using the results of Section 4 and the explicit formula (analogous to (2.10)) for the corresponding function $f\left(x_{1}, x_{2}, y(s)\right)$. We show (in Lemma 5.5) that this formula implies that

$$
\begin{gathered}
f\left(x_{1}, x_{2}, y(s)\right)=1+\left(b_{1}+o(1)\right) e^{s} e^{-i x_{1}}+\left(b_{2}+o(1)\right) e^{2 s} e^{-2 i x_{1}}+o\left(e^{2 s}\right), \text { as } s \rightarrow-\infty \\
b_{k}+o(1) \in \mathbb{R} \text { for all } s \in \mathbb{R}, k=1,2
\end{gathered}
$$

where the constants $b_{1}, b_{2}$ are expressed as independent linear and quadratic functionals in $\chi$ : their tuple is generic for a $C^{1}$-generic ${ }^{1}$ function $\chi$.

The nondifferentiability of the function $\left|f\left(x_{1}, x_{2}, y\right)\right|^{2}$ comes from the fact that the multiplier $v^{\prime}(0)=3$ of the field $v$ is greater than the module 1 of the degree of the harmonic $e^{-i x_{1}}$ in formula (5.1) defining the family of complex structures. Namely, the variable change $y \mapsto s$ can be normalized to have the asymptotics

$$
y=e^{v^{\prime}(0) s}(1+o(1)), \text { as } s \rightarrow-\infty .
$$

Indeed, the field $v(y)$ can be locally linearized at 0 by the classical linearization theorem (see $[\mathrm{ArI}]$ ), and the local (normalized) linearizing mapping extends up to a global linearizing $C^{\infty}$ mapping $h: y \in[0, \pi) \mapsto y^{\prime} \in[0,+\infty), h^{\prime}(0)=1$. In the linearized chart $y^{\prime}=e^{v^{\prime}(0) s}$, which proves (5.4). This together with formula (5.3)

\footnotetext{
1 Everywhere below by $C^{1}$-generic we mean "belonging to an open dense subset in the $C^{1}$ topology in the space of $C^{\infty}$ functions $\chi$ on $[0, \pi]$ satisfying (5.1)"
} 
implies that $f\left(x_{1}, x_{2}, y\right)=1+y^{\frac{1}{v^{\prime}(0)}} e^{-i x_{1}}\left(b_{1}+o(1)\right)+y^{\frac{2}{v^{\prime}(0)}} e^{-2 i x_{1}}\left(b_{2}+o(1)\right)$, as $y \rightarrow 0_{+}$. In our case the first power of $y$ is $y^{\frac{1}{3}}$, the second one is $y^{\frac{2}{3}}$ so, $f(x)$ is not differentiable in $y$ at $y=0$, if $b_{1} \neq 0$. The previous formula and the fact that the functional coefficients $b_{k}+o(1)$ are real imply that

$\left|f\left(x_{1}, x_{2}, y\right)\right|^{2}=1+2\left(b_{1}+o(1)\right) y^{\frac{1}{3}} \cos x_{1}+\left(b_{1}^{2}+2 b_{2} \cos \left(2 x_{1}\right)\right) y^{\frac{2}{3}}+o\left(y^{\frac{2}{3}}\right)$, as $y \rightarrow 0$.

The right-hand side of the previous formula is not differentiable in $y$ at the torus $y=0$, whenever $\chi$ is generic in the sense that $b_{1} \neq 0, b_{1}^{2}-2 b_{2} \neq 0$. Indeed, at each point of the torus where $x_{1} \neq \pi \pm \frac{\pi}{2}$ (i.e., $\cos x_{1} \neq 0$ ) the main nonconstant asymptotic term in the right-hand side is $2 b_{1} y^{\frac{1}{3}} \cos x_{1}$. At each other point, where this term vanishes $\left(\cos x_{1}=0\right.$, then $\left.\cos \left(2 x_{1}\right)=-1\right)$ the main nonconstant asymptotic term along the plane $x_{1}=$ const $=\pi \pm \frac{\pi}{2}$ is $\left(b_{1}^{2}-2 b_{2}\right) y^{\frac{2}{3}}$. This proves the nondifferentiability.

The unique family of uniformizing differentials on the leaves will be chosen normalized as follows.

5.2. Definition. Let $S$ be a cylinder, $S \rightarrow \mathbb{T}^{2}$ be a topological covering such that the liftings to $S$ of the circles in $\mathbb{T}^{2}$ tangent to the vector field $\frac{\partial}{\partial x_{1}}$ are closed curves 1-to-1 projected to the circles, $\sigma$ be a parabolic complex structure on $S$. A corresponding uniformizing differential is said to be normalized, if its integrals over the previous liftings (oriented by the vector field) are equal to $2 \pi$. A corresponding uniformizing metric is said to be normalized, if the minimal length of a loop isotopic to the previous liftings is equal to $2 \pi$. (In other terms, it is the squared module of the normalized uniformizing differential.)

5.3. Remark. In Definition 5.2 the surface $S$ is fibered by the liftings of the circles and the latters are not contractible (in particular the integrals of a uniformizing differential along them (which are equal to each other) are nonzero). The normalized uniformizing differential (metric) is well-defined and is unique. The corresponding loop of minimal length from the previous definition is a closed geodesic.

As it is shown below, Theorem 1.25 is implied by the previous discussion and the two following lemmas.

5.4. Lemma. Let $F$ be a two-dimensional foliation on a direct product $\mathbb{T}^{2} \times M$, $M$ be a manifold, $F$ be tangent to the vector field $\frac{\partial}{\partial x_{1}}$. Let the leaves of $F$ be locally 1-to-1 projected to $\mathbb{T}^{2}$ and be equipped with a $C^{\infty}$ family of $C^{\infty}$ complex structures having a uniformly bounded dilatation with respect to the standard one (lifted from $\mathbb{T}^{2}$ ). Then the corresponding normalized uniformizing differentials (metrics, see Definition 5.2) form a continuous family. If the foliation under consideration is $F_{v}, v$ is a $C^{\infty}$ vector field on $[0, \pi]$ with singularities exactly at $0, \pi$, then this is a unique continuous family of uniformizing differentials (metrics) 
up to multiplication by constant, and it is $C^{\infty}$ in the interior $\mathbb{T}^{2} \times(0, \pi)$ of the foliated space; it is analytic in the interior, if so are $v$ and the function $\mu$ defining the family $\sigma$. If $F$ is a linear foliation on $\mathbb{T}^{2} \times \mathbb{R}$ and the function $\mu$ defining $\sigma$ is $C_{b}^{\infty}$ (but now no inequality on $\|\mu\|_{C^{3}}$ is assumed, in difference to Theorem 1.23), then the corresponding family of normalized uniformizing differentials (metrics) is also $C_{b}^{\infty}$. If in the previous case of linear foliation the function $\mu$ extends holomorphically to a complex neighborhood $\left.\left|\operatorname{Im} x_{1}\right|+\left|\operatorname{Im} x_{2}\right|+|\operatorname{Im} s|<\Delta, \Delta\right\rangle 0$, of the real space $\mathbb{T}^{2} \times \mathbb{R}$ ( $s$ is a coordinate on $\mathbb{R}$ ) so that $\sup |\mu|<1$, then the corresponding family of normalized uniformizing differentials is analytic.

Lemma 5.4 is proved in 5.4 .

5.5. Lemma. Let $F$ be the standard linear foliation (5.2) on $\mathbb{T}^{2} \times \mathbb{R}$ with the coordinates $\left(x_{1}, x_{2}, s\right), \chi(s): \mathbb{R} \rightarrow \mathbb{C}$ be a $C_{b}^{\infty}$ function such that

$$
\sup |\chi|<1, \chi(s)=O\left(e^{\gamma s}\right) \text {, as } s \rightarrow-\infty ; \gamma>1, \mu\left(x_{1}, x_{2}, s\right)=\chi(s) e^{-i x_{1}} \text {. }
$$

Let $f\left(x_{1}, x_{2}, s\right): \mathbb{T}^{2} \times \mathbb{R} \rightarrow \mathbb{C} \backslash 0$ be the function such that the restriction to each leaf of the form $f \omega_{\mu}$ is the normalized uniformizing differential (see the previous definition). Then for any $k \in \mathbb{N}$

$$
f\left(x_{1}, x_{2}, s\right)=1+\sum_{j=1}^{k} g_{k}(s) e^{-i k x_{1}}+o\left(e^{k s}\right), g_{j}(s)=\left(b_{j}+o(1)\right) e^{j s}, \text { as } s \rightarrow-\infty,
$$

where $b_{j}$ are constants depending on the function $\chi$. The functions $g_{j}$ are realvalued, if so is $\chi$.

Addendum to Lemma 5.5. In the conditions of Lemma 5.5

$$
b_{1}=2 \int_{-\infty}^{+\infty} \chi(s) e^{-s} d s, b_{2}=b_{1}^{2}-4 \int_{-\infty}^{+\infty}(\chi(s))^{2} e^{-2 s} d s .
$$

5.6. Example. Let $\chi(y):[0, \pi] \rightarrow \mathbb{R}$ be a function as in $(5.1), v(y)$ be a $C^{\infty}$ vector field on $[0, \pi]$ with singularities 0 and $\pi$ that is positively directed on the interval $(0, \pi)$. Let $s:(0, \pi) \rightarrow \mathbb{R}$ be the corresponding time. Then the function $\chi(s)=\chi(y(s))$ satisfies the asymptotic condition (5.5) of the previous lemma, if $v^{\prime}(0)>1: \chi(y(s))=O(y(s))=O\left(e^{v^{\prime}(0) s}\right)$, as $s \rightarrow-\infty$.

5.7. Remark. The integrals in the Addendum converge by (5.5).

Lemma 5.5 is proved in Subsection 5.2. The Addendum is proved in Subsection 5.3.

Proof of Theorem 1.25 modulo Lemmas 5.4, 5.5 and the Addendum. Let $\chi(y)$ be a function as in (5.1). The corresponding family of complex structures on 
the leaves of $F_{v}, v(y)=3 \sin y$, has a unique continuous family of uniformizing metrics (up to constant), which can be chosen normalized (Lemma 5.4). Let us show that for appropriate $\chi$ the family of normalized uniformizing metrics is not differentiable in $y$ at the torus $y=0$. Let $s$ be the time corresponding to the vector field $v(y)=3 \sin y$. The function $\chi(y(s))$ satisfies the conditions of Lemma 5.5 (see the previous example). This together with (5.6) implies (5.3). Let $b_{1}, b_{2}$ be the constants from (5.6). Now the discussion at the beginning of the subsection proves the nondifferentiability, if $b_{1} \neq 0, b_{1}^{2}-2 b_{2} \neq 0$. These inequalities hold for a $C^{1}$-generic function $\chi(y)$ (see Footnote 1). Indeed, consider the space of $C^{1}$ functions $\chi(y)$ satisfying conditions (5.1). The values $b_{1}, b_{2}$ (see the Addendum) corresponding to $\chi(y(s))$ are bounded linear and quadratic functionals on this space and are independent. This implies the previous inequalities for a $C^{1}$-generic function $\chi$ and proves Theorem 1.25 for the corresponding family of complex structures, but without the possibility to choose it analytic. Now in any $C^{1}$-open subset of the space of functions $\chi(y)$ satisfying (5.1) (in particular, in the subset defined by the previous inequalities) one can always find an analytic function $\chi(y)$ that also extends analytically to $S^{1}$. This finishes the proof of Theorem 1.25 modulo the two previous lemmas and the Addendum.

\subsection{The family of uniformizing differentials. Proof of Lemma 5.5}

For the proof of (5.6) we prove an explicit formula (5.7) analogous to (2.10) for the function $f$ as an operator power series in $U \circ \mu$ applied to the constant function 1 ( $U$ is the operator defined in (4.18)). Then we analyze each its term $(U \circ \mu)^{k}$.

As it is shown below, (5.6) is implied by the two following statements.

5.8. Proposition. Let $F$ be the standard linear foliation (5.2) on $\mathbb{T}^{2} \times \mathbb{R}$. Let $\chi: \mathbb{R} \rightarrow \mathbb{C} \backslash 0$ be a $C_{b}^{\infty}$ function, $\sup |\chi|<1, \mu\left(x_{1}, x_{2}, s\right)=\chi(s) e^{-i x_{1}}$. Consider the corresponding family $\sigma$ of complex structures on the leaves and the family of normalized uniformizing differentials written as the restrictions to the leaves of a 1 -form $f(x) \omega_{\mu}, f\left(x_{1}, x_{2}, s\right): \mathbb{T}^{2} \times \mathbb{R} \rightarrow \mathbb{C} \backslash 0$. Let $U$ be the operator from Lemma 4.4 corresponding to $F$. Then

$$
f=1+U(\mu)+(U \circ \mu \circ U)(\mu)+\ldots
$$

Proof. Put $\nu=t \mu, t \in[0,1]$. Consider the variable complex structure $\sigma_{t}$ on the leaves of $F$ defined by the function $\nu$. Let $f_{t}\left(x_{1}, x_{2}, s\right)$ be the function defining the normalized uniformizing differentials $f \omega_{\nu}$ of the variable complex structure. For any $t$ small enough formula (5.7) holds with $f$ replaced by $f_{t}, \mu$ replaced by $\nu$ :

$$
f_{t}=(I d-U \circ \nu)^{-1}(1)=1+t U \circ \mu+t^{2} U \circ \mu \circ U(\mu)+\ldots
$$

Indeed, for any $t$ small enough the right-hand side of (5.8) is well-defined and belongs to all the Banach spaces $B_{r, k}$ defined in Subsection 4.1 (and hence, to 
$C_{b}^{\infty}\left(\mathbb{T}^{2} \times \mathbb{R}\right)$ ). This follows from inequality (4.28), which holds for a $C^{4}$-small function $\nu=t \mu$. This right-hand side satisfies differential equation (4.5) with unit initial condition, as in the proof of (2.10). For small $t$ the 1 -form $(I d-U \circ \nu)^{-1}(1) \omega_{\nu}$ defines a family of uniformizing differentials on the leaves with respect to the variable complex structure. Indeed, this form is holomorphic on the leaves by construction (equation (4.5)), and for small $t$ the corresponding function $(I d-U \circ \nu)^{-1}(1)$ is close to 1 in the space $B_{0,0}$, and hence is bounded away from zero. Now let us check that the uniformizing differentials thus constructed are normalized:

$$
\int_{x_{2}=\text { const }, s=\text { const }, x_{1} \in[0,2 \pi]}(I d-U \circ \nu)^{-1}(1) \omega_{\nu}=2 \pi .
$$

In our case, when $\mu\left(x_{1}, x_{2}, s\right)=\chi(s) e^{-i x_{1}}$, the Taylor coefficient at $t^{k}$ in the right-hand side of (5.8) is equal to

$$
(U \circ \mu)^{k}(1)=g_{k}(s) e^{-i k x_{1}}, g_{k} \in C_{b}^{\infty}(\mathbb{R}) .
$$

This follows from formula (4.18) for $U$. Thus, all the terms in the right-hand side of (5.8) except for 1 are negative harmonics in $x_{1}$ with coefficients as functions of $s$. Thus, the previous integral is equal to $2 \pi$, so, the differentials are normalized. Therefore, (5.8) holds for small $t$.

Now let us show that (5.8) holds for all $t \in[0,1]$ (in particular, its right-hand side is well defined for all $t \in[0,1])$. On each individual leaf the family $f_{t}(x) \omega_{\nu}$ of normalized uniformizing differentials (with respect to the variable complex structure) is holomorphic in $t,|t| \leq 1$ (hence, so is $f_{t}$ ). This follows from the AhlforsBers theorem $[\mathrm{AhB}]$ on holomorphy of the normalized quasiconformal mapping from Theorem 1.1 in the parameter of complex structure. As it was shown before, $f_{t}(x)$ coincides with the right-hand side in (5.8) for small $t$. The same equality (5.8) holds for all $t,|t| \leq 1$ by holomorphy of $f_{t}$ in $t,|t| \leq 1$ (in particular, the power series in (5.8) converges for $|t| \leq 1)$. Proposition 5.8 is proved.

5.9. Lemma. Let $\chi(s): \mathbb{R} \rightarrow \mathbb{C}, \chi \in C_{b}^{\infty}(\mathbb{R}), \mu\left(x_{1}, x_{2}, s\right)$ be functions as in (5.5). Let $F$ be the standard foliation (5.2) on $\mathbb{T}^{2} \times \mathbb{R}$. Let $U$ be the corresponding operator from Lemma $4.4, g_{k}(s)$ be the corresponding functions from (5.9). Then for any fixed $k \in \mathbb{N}$

$$
g_{k}(s)=\left(b_{k}+o(1)\right) e^{k s}, \text { as } s \rightarrow-\infty ; b_{k}=2 k \int_{-\infty}^{+\infty} \chi(\tau) g_{k-1}(\tau) e^{-k \tau} d \tau
$$

(we put $g_{0}=1$ ). There exists a $c_{1}>0$ such that for any $k \in \mathbb{N}$ and $s \leq 0$

$$
\left|g_{k}(s)\right| \leq c_{1}^{k} e^{\frac{k+1}{2} s}
$$

If the function $\chi$ is real-valued, then so are $g_{k}$.

Lemma 5.9 and the convergence of the integrals in (5.10) are proved below. 
5.10. Corollary. In the conditions of the previous lemma for any $k \in \mathbb{N}$

$$
\sum_{r=k}^{+\infty}\left|g_{r}(s)\right|=O\left(e^{k s}\right), \text { as } s \rightarrow-\infty .
$$

Proof. By (5.10), for any $k \in \mathbb{N} \sum_{r=k}^{2 k}\left|g_{r}(s)\right|=O\left(e^{k s}\right)$, as $s \rightarrow-\infty$. Let us prove the analogous estimate of the sum of the terms with indices $r>2 k$. By (5.11), for any $s \leq 0\left|g_{r}(s)\right| \leq c_{1}^{r} e^{\frac{r+1}{2} s} \leq\left(c_{1} e^{\frac{s}{2}}\right)^{r}$. Therefore, for any $s \leq 0$ such that $c_{1} e^{\frac{s}{2}}<\frac{1}{2}$

$$
\sum_{r>2 k}\left|g_{r}(s)\right|<2\left(c_{1} e^{\frac{s}{2}}\right)^{2 k+1} \leq 2 c_{1}^{2 k+1} e^{k s} .
$$

This together with the previous estimate of the sum from $k$ to $2 k$ proves Corollary 5.10 .

5.11. Remark. In fact, $\left|g_{k}(s)\right|<c^{k} k ! e^{k s}$ ( $c$ does not depend on $k, s$ ) for any $k \in \mathbb{N}$ and $s \leq 0$. This inequality follows from the proof of (5.10) presented below (the implication is not written to save the space) and will not be used further.

Proof of Lemma 5.5 modulo Lemma 5.9. By the previous proposition and lemma,

$$
f\left(x_{1}, x_{2}, s\right)=1+\sum_{j=1}^{+\infty} g_{j}(s) e^{-i j x_{1}} .
$$

This together with the previous corollary implies (5.6). The last statement of Lemma 5.9 together with (5.6) proves Lemma 5.5.

Proof of Lemma 5.9. Let $L_{a, c}, L_{a, c}^{*}$ be operators (4.16), $\left(L_{a, c}^{*}\right)^{-1}$ be the inverse operator from Lemma 4.9. According to the notations of Subsection 4.2, the number $a$ corresponding to the standard foliation $F$ is equal to $i$, and the complex index $n$ corresponding to the harmonic $e^{-i x_{1}}$ is also equal to $i$. By definition and (4.18),

$$
g_{1}(s)=-\left(\left(L_{i, i}^{*}\right)^{-1} \circ L_{i, i}\right) \chi(s), g_{k}(s)=-\left(\left(L_{i, k i}^{*}\right)^{-1} \circ L_{i, k i}\right)\left(\chi g_{k-1}\right)(s) .
$$

Let us firstly prove (5.10) by induction in $k$.

Induction base for (5.10). Let us calculate the right-hand side of the first formula in (5.12). By definition,

$$
L_{i, i}=-i L_{1,1} \text {, so, }-\left(L_{i, i}^{*}\right)^{-1} \circ L_{i, i}=\left(L_{1,1}^{*}\right)^{-1} \circ L_{1,1}=-1+2\left(L_{1,1}^{*}\right)^{-1}
$$

(as in the proof of Corollary 4.11 in Subsection 4.2). Thus,

$$
g_{1}=-\chi+2 q_{1}, q_{1}=\left(L_{1,1}^{*}\right)^{-1} \chi .
$$


Recall that $q_{1}(s)$ is the unique bounded solution of the differential equation $\dot{q}=$ $q-\chi$, as in the proof of Lemma 4.9. Therefore,

$$
\begin{gathered}
q_{1}(s)=e^{s} \int_{s}^{+\infty} \chi(\tau) e^{-\tau} d \tau, \text { hence, } \\
q_{1}(s)=e^{s}\left(\frac{b_{1}}{2}+o(1)\right), \text { as } s \rightarrow-\infty ; b_{1}=2 \int_{-\infty}^{+\infty} \chi(\tau) e^{-\tau} d \tau .
\end{gathered}
$$

By the asymptotic condition on $\chi$ from (5.5), the last integral converges and $\chi(s)=o\left(e^{s}\right)$, as $s \rightarrow-\infty$. This together with (5.13) and (5.15) implies (5.10) for $k=1$.

Induction step for (5.10). Suppose we have already proved (5.10) for all the indices less than a given $k$. Let us prove it for $k$. In general,

$$
g_{k}=-\chi g_{k-1}+2 q_{k}, q_{k}=k\left(L_{1, k}^{*}\right)^{-1}\left(\chi g_{k-1}\right)
$$

by (5.12), analogously to (5.13). Let us analyze the terms in the right-hand side of (5.16). By definition, $q_{k}$ is the unique bounded solution of the differential equation

$$
\dot{q}_{k}=k\left(q_{k}-\chi g_{k-1}\right) \text {, hence, } q_{k}(s)=k e^{k s} \int_{s}^{+\infty} \chi(\tau) g_{k-1}(\tau) e^{-k \tau} d \tau \text {. }
$$

The induction hypothesis implies that $g_{k-1}(s)=O\left(e^{(k-1) s}\right)$, as $s \rightarrow-\infty$ so the latter integral taken from $-\infty$ to $+\infty$ converges by (5.5). Hence, by $(5.17), q_{k}(s)=$ $\left(\frac{b_{k}}{2}+o(1)\right) e^{k s}$, where $b_{k}$ is given by the last formula from (5.10). By $(5.5), \chi(s)=$ $o\left(e^{s}\right)$, as $s \rightarrow-\infty$. Therefore, by the induction hypothesis, the first term in the right-hand side of $(5.16)$ is $-\chi(s) g_{k-1}(s)=o\left(e^{k s}\right)$. This together with (5.16) and the previous asymptotic formula for $q_{k}$ proves the induction step. Formula (5.10) is proved.

Now let us prove (5.11) by induction in $k$ and calculate simultaneously the corresponding constant $c_{1}$. The induction base for $k=1,2$ with appropriate constant $c_{1}$ follows from (5.10). In the proof of the induction step we use the following inequalities:

$$
\left|g_{k}\right| \leq c_{2}^{k}, c_{2}=3 \sup |\chi|
$$

$$
|\chi(s)|<c_{3} e^{\gamma s} \text { for all } s \leq 0 ; \gamma>1 \text { and } c_{3}>0 \text { do not depend on } s \text {. }
$$

Inequality (5.19) follows from (5.5). Inequality (5.18) is proved by induction in $k$. The induction base for $k=1$ follows from (5.9) and (4.11). Let us prove the induction step assuming that the inequality is already proved for all the indices less than a given $k$. To do this, we use formula (5.16) for $g_{k}$ and estimate the terms of its right-hand side. One has $\sup \left|q_{k}\right| \leq \sup \left|\chi g_{k-1}\right|$, as in Section 4 (by Lemma 4.9, $\left.\left\|\left(L_{1, k}^{*}\right)^{-1}\right\|_{C^{0}}=\frac{1}{k}\right)$. This together with (5.16) implies that $\left|g_{k}\right| \leq$ $3 \sup |\chi| \sup \left|g_{k-1}\right|$. Thus, by the induction hypothesis, $\left|g_{k}\right| \leq(3 \sup |\chi|)^{k}$. This proves the induction step. Inequality (5.18) is proved.

Induction step for (5.11). Let us prove (5.11) for a given $k \geq 3$ assuming that it is proved for all the smaller indices. To do this, we use formula (5.16) for $g_{k}$ and 
estimate the terms in its right-hand side. The first term is estimated by (5.19) and the induction hypothesis:

$$
\left|\chi(s) g_{k-1}(s)\right| \leq c_{3} e^{\gamma s} c_{1}^{k-1} e^{\frac{k}{2} s} \leq c_{3} c_{1}^{k-1} e^{\frac{k+1}{2} s} \text { for any } s \leq 0 .
$$

To prove an upper bound of the function $2 q_{k}$ (the second term), we use (5.18) and estimate the integral in the formula for $q_{k}$ from (5.17). We split it into two integrals: 1) from $s$ to $0 ; 2$ ) from 0 to $+\infty$. By (5.18), the module of the latter integral is no greater than $c_{2}^{k} \int_{0}^{+\infty} e^{-k \tau} d \tau=\frac{c_{2}^{k}}{k}$. Let us estimate the module of the former integral from $s$ to 0 . Replacing $\chi(\tau)$ by $c_{3} e^{\tau}$ (see inequality (5.19)) and $g_{k-1}(\tau)$ by $c_{1}^{k-1} e^{\frac{k}{2} \tau}$ (the induction hypothesis) does not diminish its module and transforms it to the integral

$$
c_{3} c_{1}^{k-1} \int_{s}^{0} e^{\frac{2-k}{2} \tau} d \tau=\frac{2 c_{3} c_{1}^{k-1}}{k-2}\left(e^{\frac{2-k}{2} s}-1\right) \leq \frac{6}{k} c_{3} c_{1}^{k-1} e^{\frac{2-k}{2} s} .
$$

Adding the estimates of the integrals yields that for any $s \leq 0$

$$
\left|q_{k}(s)\right| \leq k e^{k s}\left(\frac{c_{2}^{k}}{k}+\frac{6}{k} c_{3} c_{1}^{k-1} e^{\frac{2-k}{2} s}\right)=c_{2}^{k} e^{k s}+6 c_{3} c_{1}^{k-1} e^{\frac{k+2}{2} s} \leq\left(c_{2}^{k}+6 c_{3} c_{1}^{k-1}\right) e^{\frac{k+2}{2} s} .
$$

Substituting the last inequality and (5.20) to (5.16) yields that for any $s \leq 0$

$$
\left|g_{k}(s)\right| \leq c_{3} c_{1}^{k-1} e^{\frac{k+1}{2} s}+2\left(c_{2}^{k}+6 c_{3} c_{1}^{k-1}\right) e^{\frac{k+2}{2} s} \leq\left(13 c_{3} c_{1}^{k-1}+2 c_{2}^{k}\right) e^{\frac{k+1}{2} s} .
$$

Let us choose a $c_{1}$ that satisfies the induction base in such a way that $c_{1}^{k}>$ $13 c_{3} c_{1}^{k-1}+2 c_{2}^{k}$ for any $k>1$. To achieve this, it suffices to choose $c_{1}>13 c_{3}+2 c_{2}+1$. Then (5.21) implies (5.11). This proves the induction step and inequality (5.11). The last statement of Lemma 5.9 (saying that $g_{k}$ are real, if so is $\chi$ ) follows from (5.13) and (5.16). The proof of Lemma 5.9 is completed.

\subsection{Formulas for $b_{1}$ and $b_{2}$. Proof of the Addendum to Lemma 5.5}

The formula for $b_{1}$ from the Addendum follows from (5.10), which is already proved. Let us prove the formula for $b_{2}$. Formula (5.10) for $k=2$ expresses $b_{2}$ in terms of $g_{1}$. Substituting to it formulas (5.13) for $g_{1}$ and (5.14) for $q_{1}$ yields

$b_{2}=4 \int_{-\infty}^{+\infty} \chi(\tau) g_{1}(\tau) e^{-2 \tau} d \tau=4 \int_{-\infty}^{+\infty} \chi(\tau) e^{-2 \tau}\left(-\chi(\tau)+2 e^{\tau} \int_{\tau}^{+\infty} \chi(\phi) e^{-\phi} d \phi\right) d \tau$.

Denote

$$
F(\tau)=\int_{\tau}^{+\infty} \chi(\phi) e^{-\phi} d \phi ; \text { then } F(\tau) \rightarrow \int_{-\infty}^{+\infty} \chi(\phi) e^{-\phi} d \phi=\frac{b_{1}}{2}, \text { as } \tau \rightarrow-\infty .
$$

By (5.22), (5.23), one has

$$
b_{2}=-4 \int_{\infty}^{+\infty}(\chi(\tau))^{2} e^{-2 \tau} d \tau+8 \int_{-\infty}^{\infty} \chi(\tau) e^{-\tau} F(\tau) d \tau
$$


By (5.23), the second integral in the last formula is equal to

$$
-\int_{-\infty}^{+\infty} F^{\prime}(\tau) F(\tau) d \tau=-\frac{1}{2} \int_{-\infty}^{+\infty} d F^{2}(\tau)=\frac{b_{1}^{2}}{8} .
$$

Therefore,

$$
b_{2}=-4 \int_{\infty}^{+\infty}(\chi(\tau))^{2} e^{-2 \tau} d \tau+b_{1}^{2} .
$$

This proves the Addendum.

\subsection{Existence and uniqueness of continuous families of uniformizing metrics and uniformizing differentials. Proof of Lemma 5.4}

The proof of Lemma 5.4 is similar to the proof of an analogous statement from $[\mathrm{G}]$ for linear foliations of $\mathbb{T}^{3}$ by cylinders. We present a proof only for a linear foliation $F$ on $\mathbb{T}^{2} \times \mathbb{R}$ by cylinders: the proof in the general case is analogous. The uniqueness statement for foliations $F_{v}$ will be proved at the end of the subsection.

Let us reformulate the definition of normalized uniformizing differential. Each leaf $L$ of $F$ with a base point $a \in L$ and its universal covering $\widetilde{L}(a)$ can be regarded as coverings over $\mathbb{T}^{2}$ equipped with the "standard" complex structure lifted from $\mathbb{T}^{2}$. Each universal covering $\widetilde{L}(a)$ is identified canonically with $\mathbb{C}$ by composition of its projection and the inverse to the canonical projection $\mathbb{C} \rightarrow \mathbb{T}^{2}$. The "nonstandard" complex structure $\sigma$ on $L$ defined by the function $\mu$ induces a complex structure on $\widetilde{L}(a)$ (also denoted by $\sigma$ ) that is $C^{\infty}$ in the coordinates of $a$. By Theorem 1.1, there exists a quasiconformal diffeomorphism $\Phi: \widetilde{L}(a) \rightarrow \mathbb{C}$ transforming $\sigma$ to the standard complex structure.

Denote the base point of the universal covering $\widetilde{L}(a)$ by the same symbol $a$. Consider the lifting to $\widetilde{L}(a)$ starting at $a$ of the positively oriented circle in $L$ tangent to the vector field $\frac{\partial}{\partial x_{1}}$. Denote by $a^{\prime}$ the endpoint of this lifting. Let us normalize the previous quasiconformal diffeomorphism $\Phi=\Phi_{a}$ so that $\Phi_{a}(a)=0$, $\Phi_{a}\left(a^{\prime}\right)=2 \pi$ (this defines $\Phi$ uniquely). Then $d \Phi$ is the normalized uniformizing differential.

Consider $\Phi_{a}$ as a quasiconformal diffeomorphism $\mathbb{C} \rightarrow \mathbb{C}$ (recall that $\widetilde{L}(a)$ is identified canonically with $\mathbb{C}$ ). Then $\Phi_{a}$ is $C_{b}^{\infty}$ in the coordinates of the base point $a$, if the function $\mu$ defining the family of complex structures is $C_{b}^{\infty}\left(\mathbb{T}^{2} \times \mathbb{R}\right)$. The diffeomorphism $\Phi_{a}$ is analytic in $a$, if $\mu$ extends holomorphically to a complex neighborhood $\left.\left|\operatorname{Im} x_{1}\right|+\left|\operatorname{Im} x_{2}\right|+|\operatorname{Im} s|<\Delta, \Delta\right\rangle 0$, of the real foliated space. Both statements follow from the Ahlfors-Bers theorem [AhB]. This implies that the family of normalized uniformizing differentials is $C_{b}^{\infty}$ (analytic in the latter case) and proves the statement of Lemma 5.4 concerning linear foliations by cylinders.

Let us prove the uniqueness statement of Lemma 5.4 for a foliation $F_{v}$ : the uniqueness of a continuous family of uniformizing differentials up to multiplication by constant. The proof of uniqueness of a continuous family of uniformizing metrics 
is analogous. For a given uniformizing differential on a toric leaf $L^{\prime}$ (defined uniquely up to multiplication by constant) and any noncompact leaf $L$ approaching $L^{\prime}$ there is at most a unique uniformizing differential on $L$ approaching the given one on $L^{\prime}$. Each noncompact leaf accumulates to any toric leaf. This implies the uniqueness and proves Lemma 5.4.

\section{Unbounded counterexample. Proof of Theorem 1.27}

\subsection{Motivations of the construction of the counterexample and the scheme of the proof of Theorem 1.27}

6.1. Definition. A $C^{\infty}$ complex structure on $\mathbb{R}^{2}$ is said to be rotationally-orthogonal, if it is invariant under rotations fixing 0 and the circles centered at 0 are orthogonal to the radial rays going from 0 with respect to the complex structure. We say that a complex structure on $\mathbb{R}^{2}$ tends to the standard complex structure at infinity, if $\mu(x) \rightarrow 0$, as $x \rightarrow \infty$ ( $\mu$ is the corresponding function from (1.1)).

The starting point of the construction of a foliation from Theorem 1.27 with unbounded uniformizing differentials is the following

6.2. Lemma. There exists a rotationally-orthogonal $C^{\infty}$ complex structure on $\mathbb{R}^{2}$ tending to the standard complex structure at infinity such that the corresponding uniformizing differential is an unbounded differential 1-form on $\mathbb{R}^{2}$ : it tends to infinity, as the point where it is taken tends to infinity.

A stronger statement (Lemma 6.4 below) will be proved in the next subsection.

Lemma 6.2 allows us to construct an abstract lamination of a compact topological space by two leaves $\left(\mathbb{T}^{2}\right.$ and $\mathbb{C}$ ) admitting two continuous families of complex structures with the following properties. For each one of these families the leaves are conformally equivalent respectively to $\mathbb{T}^{2}$ and $\mathbb{C}$. The first complex structure (called "standard") admits a continuous family of uniformizing differentials and this is not true for the second one (the leaf $\mathbb{C}$ has an unbounded uniformizing differential).

Namely, consider the standard coordinate $x_{1}, x_{2}$-plane $L_{0}$ in $\mathbb{R}^{3}$ (with the coordinates $x_{1}, x_{2}, x_{3}$, the complex coordinate $z=x_{1}+i x_{2}$ defines the standard complex structure on it). Consider its abstract copy $L_{1}$ that is embedded to $\mathbb{R}^{3}$ as a graph of a function $x_{3}=F\left(x_{1}, x_{2}\right)$, where $F\left(x_{1}, x_{2}\right) \rightarrow 0$, as $\left(x_{1}, x_{2}\right) \rightarrow \infty$. We assume that the graph $L_{1}$ is equipped with the coordinates $\left(x_{1}, x_{2}\right) \in \mathbb{R}^{2}$. We equip $L_{0}$ with the standard complex structure and $L_{1}$ with the complex structure from Lemma 6.2. Take the union $M=L_{0} \cup L_{1} \subset \mathbb{R}^{3}$. This union is laminated by two leaves but it is not compact. It is a metric space with the metric inherited from $\mathbb{R}^{3}$. To construct a lamination of a compact space, consider the standard 
projection $p: \mathbb{R}^{2}=L_{0} \rightarrow \mathbb{T}^{2}$. Consider the equivalence relation on $M$ saying that any two $p$-preimages of each point in $L_{0}$ are equivalent. Denote the corresponding quotient space by $M^{\prime}$. The space $M^{\prime}$ admits a natural structure of metric space and is compact: the distance between any two points is defined to be the minimal distance between their preimages in $M$. The space $M^{\prime}$ is laminated by two surfaces: one of them is $\mathbb{T}^{2}=L_{0} / p$, the other one is identified with $L_{1}$ and will be denoted by the same symbol $L_{1}$. The leaves carry complex structures defined above (denote them by $\sigma$ ).

The function $\mu$ defining $\sigma$ is continuous on $M^{\prime}$. On the other hand, the uniformizing differential of the leaf $L_{1}$ is unbounded by Lemma 6.2.

6.3. Remark. It is not known whether one can embed the previous lamination $M^{\prime}$ to a $C^{\infty}$ foliation on a compact manifold in such a way that $\sigma$ extends up to a $C^{\infty}$ family of complex structures on the leaves of the foliation (for appropriately chosen complex structure from Lemma 6.2 on the leaf $L_{1}$ ).

Now let us modify the previous lamination $M^{\prime}$ in order to make it embedded as above (the ambient foliated manifold will be $\mathbb{T}^{2} \times S^{2}$ ). Let

$$
D_{1}=\{|z|<1\} \subset \overline{\mathbb{C}}=S^{2}, S^{1}=\partial D_{1} .
$$

Consider a diffeomorphism $h: \mathbb{R}^{2} \rightarrow D_{1}$ preserving the radial rays and commuting with the rotations centered at 0 : more precisely, taking the form

$$
h:(r, \phi) \mapsto(R(r), \phi) .
$$

in the polar coordinates $(r, \phi)$, where $R:[0,+\infty) \rightarrow[0,1)$ is a one-dimensional diffeomorphism. This yields the embedding

$$
H=p \times h: \mathbb{R}^{2} \rightarrow L_{h} \subset \mathbb{T}^{2} \times D_{1} \subset \mathbb{T}^{2} \times S^{2}, L_{h}=H\left(\mathbb{R}^{2}\right) .
$$

The image $L_{h}$ will be playing the role of $L_{1}$ : we take the complex structure on $L_{h}$ that is the pushforward under $H$ of a complex structure from Lemma 6.2. Take the standard complex structure on the fibers $\mathbb{T}^{2} \times \phi, \phi \in S^{1}$. The lamination consisting of the latter fibers and $L_{h}$ extends up to the foliation on $\mathbb{T}^{2} \times \overline{D_{1}}$ defined by the splitting

$$
\mathbb{T}^{2} \times \overline{D_{1}}=\bigcup_{\phi \in S^{1}}\left(\mathbb{T}^{2} \times \phi\right) \cup \bigcup_{\tau \in \mathbb{T}^{2}}\left(L_{h}+(\tau, 0)\right) .
$$

Denote the foliation thus obtained by $F_{h}$. It is extended by the symmetry with respect to $S^{1}$ up to a foliation on $\mathbb{T}^{2} \times S^{2}$ denoted by the same symbol $F_{h}$. We show that for appropriately chosen $h$ and the initial complex structure on $L_{h}$ the foliation by Riemann surfaces thus obtained satisfies the statements of Theorem 1.27.

Theorem 1.27 (and Lemma 6.2) are implied by the following

6.4. Lemma. There exist a rotationally-orthogonal complex structure $\sigma$ on $\mathbb{R}^{2}$ tending to the standard one at infinity (see Definition 6.1) and a $C^{\infty}$ function 
$R(r):[0,+\infty) \rightarrow[0,1), R^{\prime}>0, R(r) \rightarrow 1$, as $r \rightarrow+\infty$, that satisfy the following statements:

1) The uniformizing differential of $\sigma$ is unbounded and tends to infinity, as the point where it is taken tends to infinity.

2) Let $h$ be the diffeomorphism (6.1), $H: \mathbb{R}^{2} \rightarrow L_{h} \subset \mathbb{T}^{2} \times \overline{D_{1}}, F_{h}$ be respectively the corresponding embedding (6.2) and foliation (6.3). Let now $R$ be considered as the radial polar coordinate on $D_{1}$. The foliation $F_{h}$ is $C^{\infty}$ on $\mathbb{T}^{2} \times \overline{D_{1}}$ and flat in the coordinate $R$ at the boundary $\mathbb{T}^{2} \times S^{1}$ (i.e., the corresponding tangent plane field has all the derivatives in $R$ zero at the boundary).

3) Consider the family of complex structures on the leaves of $F_{h}$ defined on $L_{h}$ as the pushforward of $\sigma$ by $H$, its translation pushforwards on the other noncompact leaves $L_{h}+(\tau, 0)$ and the standard complex structure on the toric leaves in $\mathbb{T}^{2} \times S^{1}$. This family is $C^{\infty}$ and it is flat in the polar coordinate $R$ at the boundary $\mathbb{T}^{2} \times S^{1}$.

Lemma 6.4 is proved in the next subsection.

Proof of Theorem 1.27 modulo Lemma 6.4. Let $F_{h}$ be the foliation from Lemma 6.4. It extends up to a $C^{\infty}$ foliation on $\mathbb{T}^{2} \times S^{2}$ by the symmetry with respect to $S^{1}$ (flatness, see statement 2) of Lemma 6.4). This foliation satisfies the statements 1)-4) of Theorem 1.27 by construction. The family of complex structures from statement 3) of Lemma 6.4 extends analogously (flatness) up to a $C^{\infty}$ family of complex structures on all the leaves in $\mathbb{T}^{2} \times S^{2}$. The uniformizing metric of each noncompact leaf tends to infinity, as the point where it is taken tends to $\mathbb{T}^{2} \times S^{1}$ (statement 1) of Lemma 6.4). This proves Theorem 1.27.

In the proof of Lemma 6.4 we use the following properties of a rotationallyorthogonal complex structure and formula for the corresponding uniformizing differential.

6.5. Remark. Let $r, \phi$ be the polar coordinates on $\mathbb{R}^{2}$. Then a complex structure on $\mathbb{R}^{2}$ is rotationally-orthogonal, if and only if it admits a $\mathbb{C}$-linear differential of the type

$$
\omega_{k}=e^{i \phi}(d r+i r k(r) d \phi), k:[0,+\infty) \rightarrow(0,+\infty) .
$$

(The corresponding function $k$ is uniquely defined.) The complex structure is standard at a given point $a \in \mathbb{R}^{2}$, if and only if $k(a)=1$. If

$$
k \in C^{\infty}\left(\mathbb{R}_{+} \cup 0\right), k \equiv 1 \text { near zero, } k \text { is bounded away from } 0 \text { and } \infty,
$$

then the corresponding complex structure is $C^{\infty}$ and has a bounded dilatation; hence it is parabolic, i.e., isomorphic to $\mathbb{C}$ (Theorem 1.1).

6.6. Proposition. Let $k$ be a real function as in (6.5), $\omega_{k}$ be the corresponding 1-form (6.4). Then the uniformizing differential of the corresponding (parabolic) 
complex structure on $\mathbb{R}^{2}$ has the form $f(r) \omega_{k}$, where $f(r)>0$,

$$
\ln f(r)=\int_{0}^{r} \frac{d s}{s k(s)}-\ln r-\ln k(r) .
$$

Proposition 6.6 is proved in 6.3 .

\subsection{Unbounded uniformizing differential. Proof of Lemma 6.4}

Take a function $k \in C^{\infty}[0,+\infty)$ such that

$$
k(r)>0,\left.k(r)\right|_{r<1} \equiv 1,\left.k\right|_{r>4}=1-\frac{1}{\ln r} .
$$

Take $R(r)$ to be a $C^{\infty}$ function with the following properties:

$$
R^{\prime}>0,\left.R\right|_{0 \leq r \leq \frac{1}{4}} \equiv r ; R_{r>3^{9}} \equiv 1-\frac{1}{\ln \ln r} .
$$

(A $C^{\infty}$ function $R$ satisfying (6.8) exists, since its value $\frac{1}{4}$ at the end of the former segment $\left[0, \frac{1}{4}\right]$ is less than its value $1-\frac{1}{\ln \ln 3^{9}}>\frac{1}{2}$ at the starting point of the latter semiinterval $\left[3^{9},+\infty\right)$.) Below we show that the complex structure on $\mathbb{R}^{2}$ defined by the function $k(r)$ from (6.7) (see (6.4)) and the function $R$ from (6.8) satisfy the statements of Lemma 6.4.

Let us prove statement 1) of Lemma 6.4: the uniformizing differential tends to infinity. Let $f(r)$ be the function from (6.6). By Proposition 6.6, the previous statement is equivalent to say that $f(r) \rightarrow+\infty$, as $r \rightarrow+\infty$, or so does $\ln f$. When $r>4$, the integral in (6.6) is equal to

$$
\begin{gathered}
\text { const }+\int_{4}^{r} \frac{d s}{s\left(1-(\ln s)^{-1}\right)}=\mathrm{const}+\int_{4}^{r} \frac{d \ln s}{1-(\ln s)^{-1}}= \\
\text { const }+\int_{4}^{r}\left(1+(\ln s)^{-1}+O\left((\ln s)^{-2}\right)\right) d \ln s=\ln r+\ln \ln r+O(1),
\end{gathered}
$$

as $r \rightarrow+\infty$. This together with (6.6) and boundedness of $\ln k(r)$ implies that the right-hand side of (6.6) is $\ln \ln r+O(1) \rightarrow+\infty$, as $r \rightarrow+\infty$, thus, $\ln f(r) \rightarrow+\infty$. This proves statement 1) of Lemma 6.4.

Now let us prove statement 2) of Lemma 6.4. The corresponding mapping $h$ from (6.1) is a diffeomorphism by definition. Statement 2) of Lemma 6.4 is implied by the following

6.7. Proposition. Let $R(r)$ be a $C^{\infty}$ function as in (6.8), $h: \mathbb{R}^{2} \rightarrow D_{1}$ be the corresponding diffeomorphism (6.1), $H, L_{h}$ be respectively the corresponding embedding of $\mathbb{R}^{2}$ and its image (6.2), $F_{h}$ be the corresponding foliation (6.3) on $\mathbb{T}^{2} \times \overline{D_{1}}$. The foliation $F_{h}$ is $C^{\infty}$ on $\mathbb{T}^{2} \times \overline{D_{1}}$ and flat in the radial polar coordinate of $D_{1}$ at $\mathbb{T}^{2} \times S^{1}$. 
Proof. Let $(R, \theta)$ be the polar coordinates on $D_{1}$. The foliation $F_{h}$ is $C^{\infty}$ in the interior $\mathbb{T}^{2} \times D_{1}$, by definition and since so is $h$. Let us prove that it is flat in $R$ and $C^{\infty}$ at $\mathbb{T}^{2} \times S^{1}$. Let $\tau=\left(\tau_{1}, \tau_{2}\right)$ be the affine coordinates on $\mathbb{T}^{2}$. Denote $a_{R, \theta}=(0, R, \theta) \in \mathbb{T}^{2} \times \overline{D_{1}}, 0<R \leq 1$ (the coordinates $\tau$ of $a_{R, \theta}$ are zeroes). It suffices to prove flatness in $R$ and smoothness of the foliation $F_{h}$ at arbitrary point $a_{1, \theta}=(0,1, \theta) \in \mathbb{T}^{2} \times S^{1}$. Let us calculate the slope at $a_{R, \theta}$ of the corresponding tangent plane to $F_{h}$. Let $(r, \phi)$ be the polar coordinates on $\mathbb{R}^{2}$, which is the source for the embedding $H: \mathbb{R}^{2} \rightarrow \mathbb{T}^{2} \times \overline{D_{1}}$. By definition, $a_{R, \theta} \in H\left(\mathbb{R}^{2}\right)+(\tau, 0)$, $\tau \in \mathbb{T}^{2}$. Denote $a_{R, \theta}^{\prime}=H^{-1}\left(a_{R, \theta}-(\tau, 0)\right)$. Denote by $r(R)$ the function inverse to the function $R(r)$, see (6.8). By $(6.1),(6,2)$,

$$
a_{R, \theta}^{\prime}=(r(R), \theta) \text { in the polar coordinates. }
$$

Consider the polar coordinate unit tangent vectors $\frac{\partial}{\partial r}, r^{-1} \frac{\partial}{\partial \phi}$ attached at $a_{R, \theta}^{\prime}$ and their images under the previous embedding $H+(\tau, 0)$ (thus attached at $a_{R, \theta}$ ). Denote their images by $v_{1}(R, \theta)$ and $v_{2}(R, \theta)$ respectively. By definition, they are tangent to the foliation $F_{h}$. By $(6.1),(6.2)$, their components in the coordinates $\left(\tau_{1}, \tau_{2}, R, \theta\right)$ are equal to

$$
v_{1}(R, \theta)=\left(\cos \theta, \sin \theta, R^{\prime}(r(R)), 0\right), v_{2}(R, \theta)=\left(-\sin \theta, \cos \theta, 0,(r(R))^{-1}\right) .
$$

Thus, the $\tau$-components of the latter vectors depend only on $\theta$ and are linearly independent. Now to prove that the tangent plane field corresponding to the foliation $F_{h}$ is flat in $R$ and $C^{\infty}$ at $\mathbb{T}^{2} \times S^{1}$, it suffices to show that the $(R, \theta)$ components of the vectors (6.9) (which depend only on $R$ ) extend up to $C^{\infty}$ functions in $R$ flat at the point $R=1$. Recall that by $(6.8), R(r)=1-\frac{1}{\ln \ln r}$, when $r$ is large (equivalently, when $R$ is close to 1 ). Therefore,

$$
\ln r(R)=e^{\frac{1}{1-R}}, R^{\prime}(r)=\frac{1}{r \ln r(\ln \ln r)^{2}}=(1-R)^{2} e^{-\frac{1}{1-R}} \exp \left(-e^{\frac{1}{1-R}}\right) .
$$

Formula (6.10) implies that the $(R, \theta)$ components of the vectors $(6.9)$ are $C^{\infty}$ as functions in $R \in(0,1]$ and flat at $R=1$. Proposition 6.7 is proved.

Now let us prove statement 3) of Lemma 6.4: smoothness and flatness of the family of complex structures at $\mathbb{T}^{2} \times S^{1}$. This family is $C^{\infty}$ a priori in the interior $\mathbb{T}^{2} \times D_{1}$. To show that it is flat in $R$ and $C^{\infty}$ at $\mathbb{T}^{2} \times S^{1}$, it suffices to prove the same statement for the function $k(r(R))$. But this statement follows from (6.7) and the first formula in (6.10). Lemma 6.4 is proved.

\subsection{Proof of Proposition 6.1}

A form $f(r) \omega_{k}$ is closed, if and only if the function $f(r)$ satisfies the differential equation

$$
\frac{d(f(r) k(r) r)}{d r}=f(r)
$$


Denote $F(r)=f(r) k(r) r$. By $(6.11)$,

$$
F^{\prime}(r)=(r k(r))^{-1} F .
$$

This implies (6.6), and vice versa, (6.6) implies the closeness of the form $f \omega_{k}$. Consider the mapping $\Phi: z \mapsto \int_{0}^{z} f \omega_{k}$, which is well-defined on $\mathbb{R}^{2}$ (by closedness of the form), transforms the complex structure defined by $\omega_{k}$ to the standard one and has the derivative equal to $f \omega_{k}$. In particular, $\Phi$ is a local diffeomorphism. To show that the derivative $f \omega_{k}$ is a uniformizing differential, it suffices to prove that $\Phi$ is a diffeomorphism onto $\mathbb{C}$. Indeed, $\Phi$ is rotation-invariant and maps the radial rays going from 0 to the ones with the same azimuths by construction. Hence, it is a diffeomorphism of the plane $\mathbb{R}^{2}$ onto a domain in $\mathbb{C}$. The latter is rotationinvariant (by the same property of $\Phi$ ), and hence, it is either a disc, or the whole $\mathbb{C}$. The first case is impossible, since in this case the plane $\mathbb{R}^{2}$ equipped with the complex structure defined by $k$ would be conformally equivalent to a disc, which would contradict the parabolicity (the last statement of Remark 6.5). Hence, $\Phi$ is a diffeomorphism onto $\mathbb{C}$. Proposition 6.1 is proved.

Acknowledgements. I am grateful to Yu. S. Ilyashenko and É. Ghys, who have attracted my attention to the problem. I am grateful to them and to É. Giroux, J. Milnor, J.-P. Otal for helpful discussions. I wish to thank the referee for a lot of work in reading the paper. His very helpful and detailed remarks improved the paper very much. Research was supported by part by CRDF grant RM1-2358MO-02, by Russian Foundation for Basic Research (RFBR) grant 02-01-00482, by State Scientific Fellowship of Russian Academy of Sciences for young scientists, by the European Post-Doctoral Institute joint fellowship of Max-Planck Institut für Mathematik (Bonn) and IHÉS (Bures-sur-Yvette, France). The paper was written in part during my stay at the Independent University of Moscow and IHÉS that was supported by the latter fellowship. I wish to thank all these Institutions for hospitality and support.

\section{References}

[Ab] W. Abikoff, Real analytic theory of Teichmüller space, Lect. Notes in Math., 820, Springer-Verlag, 1980.

[Ah1] L. Ahlfors, Conformality with respect to Riemannian metrics, Ann. Acad. Sci. Fenn. Ser. A. I. 1955 (1955), no. 206, 22 pp.

[Ah2] L. Ahlfors, Lectures on quasiconformal mappings, Wadsworth, 1987.

[AhB] L. Ahlfors and L. Bers, Riemann's mapping theorem for variable metrics, Ann. of Math. (2) 72 (1960), 385-404.

[Ar] V. I. Arnold, Ordinary differential equations (in Russian), Third edition, Nauka, Moscow, 1984. 272 pp. Translated to English by Roger Cooke, Springer Textbook, Springer-Verlag, Berlin, 1992. 334 pp. 
[ArI] V. I. Arnold and Yu. S. Ilyashenko, Ordinary Differential Equations, in: Dynamical Systems - 1, Itogi Nauki i Techniki, Contemporary Problems in Mathematics, Fundamental Directions, VINITI, Moscow, 1985, vol. 1. English translation in: Encyclopaedia Math. Sci., 1, Dynamical systems, I, 1-148, Springer, Berlin, 1988.

[Ca] A. Candel, Uniformization of surface laminations, Ann. Sci. École Norm. Sup. (4) 26 (1993), no. 4, 489-516.

[CGM] A. Candel and X. Gómez-Mont, Uniformization of the leaves of a rational vector field, Ann. Inst. Fourier (Grenoble) 45 (1995), no. 4, 1123-1133.

[Ch] Y. Choquet-Bruhat, C. de Witt-Morette and M. Dillard-Bleick, Analysis, Manifolds and Physics, North-Holland, 1977.

[DB] A. Douady et X. Buff, Le théorème d'intégrabilité des structures prèsque complexes (French), Integrability theorem for almost complex structures, in: The Mandelbrot set, theme and variations, 307-324, London Math. Soc. Lecture Note Ser., 274, Cambridge Univ. Press, Cambridge, 2000.

[G] É. Ghys, Sur l'uniformisation des laminations paraboliques, in: Integrable systems and foliations, ed. C. Albert, R. Brouzet, J.-P. Dufour (Montpellier, 1995), Progress in Math. 145 (1996), 73-91.

[Gl] A. Glutsyuk, Simple proofs of uniformization theorems, to appear.

[H] A. Haefliger, Some remarks on foliations with minimal leaves, J. Differential Geometry 15 (1980), no 2, 269-284.

[Ko] A. Korn, Zwei Anwendungen der Methode der sukzessiven Annäherungen, in: Schwarz Festschrift, Berlin, 1919, pp. 215-229.

[La] M. A. Lavrentiev, Sur une classe des représentations continues, Mat. Sb. 42 (1935), 407434.

[Li1] A. Lins Neto, Simultaneous uniformization for the leaves of projective foliations by curves, Bol. Soc. Brasil. Mat. (N.S.) 25 (1994), no. 2, 181-206.

[Li2] A. Lins Neto, Uniformization and the Poincaré metric on the leaves of a foliation by curves, Bol. Soc. Brasil. Mat. (N.S.) 31 (2000), no. 3, 351-366.

[Licht] L. Lichtenstein, Zur Theorie der konformen Abbildungen; Konforme Abbildungen nichtanalytischer singularitätenfreier Flächenstücke auf ebene Gebiete, Bull. Acad. Sci. Cracovie (1916), 192-217.

[M] C. B. Morrey, Jr., On the solutions of quasi-linear elliptic partial differential equations, Trans. Amer. Math. Soc. 43 (1938), no. 1, 126-166.

[Vek] I. N. Vekua, The problem of reduction to canonical form of differential forms of elliptic type and the generalized Cauchy-Riemann system (Russian), Dokl. Akad. Nauk SSSR (N.S.) 100 (1955), 197-200.

[Ver] A. Verjovsky, A uniformization theorem for holomorphic foliations, in: The Lefschetz centennial conference, Part III (Mexico City, 1984), 233-253, Contemp. Math., 58, III, Amer. Math. Soc., Providence, RI, 1987.

\author{
A. A. Glutsyuk \\ CNRS \\ Unité de Mathématiques Pures et Appliquées, M.R. \\ École Normale Supérieure de Lyon \\ 46 allée d'Italie \\ 69364 Lyon Cedex 07 \\ France \\ e-mail: aglutsyu@umpa.ens-lyon.fr
}

(Received: February 21, 2000; revised version: February 10, 2003) 